\title{
The Hubble Space Telescope Advanced Camera for Surveys Emission Line Survey of Andromeda. I. Classical Be Stars
}

\author{
M. Peters ${ }^{1}$ (D) , J. P. Wisniewski ${ }^{1}$ (D), B. F. Williams ${ }^{2}$ (D), J. R. Lomax $^{3}$ (D), Y. Choi ${ }^{4}$, M. Durbin ${ }^{2}$, L. C. Johnson ${ }^{5}$ (D) A. R. Lewis ${ }^{6,7}$, \\ J. Lutz ${ }^{2}$, T. A. A. Sigut ${ }^{8}$ (D), A. Wallach ${ }^{2}$, and J. J. Dalcanton ${ }^{2}$ (i) \\ ${ }^{1}$ Homer L. Dodge Department of Physics, University of Oklahoma, 440 W. Brooks St., Norman, OK 73071, USA \\ ${ }^{2}$ Department of Astronomy, University of Washington, Box 351580, Seattle, WA 98195, USA \\ ${ }^{3}$ United States Naval Academy, Physics Department, 572 C Holloway Rd., Annapolis, MD, 21402, USA \\ ${ }^{4}$ Steward Observatory, University of Arizona, 933 North Cherry Ave., Tucson AZ 85721, USA \\ ${ }^{5}$ Department of Physics and Astronomy, Northwestern University, 2145 Sheridan Rd., Evanston, IL 60208, USA \\ ${ }_{7}^{6}$ Center for Cosmology and AstroParticle Physics, The Ohio State University, Columbus, OH 43210, USA \\ ${ }^{7}$ Department of Astronomy, The Ohio State University, 140 West 18th Ave., Columbus, OH 43210, USA \\ ${ }^{8}$ Department of Physics and Astronomy, University of Western Ontario, London, ON, N6A 3K7, Canada \\ Received 2019 August 12; revised 2020 January 7; accepted 2020 January 8; published 2020 February 21
}

\begin{abstract}
We present results from a two epoch Hubble Space Telescope $\mathrm{H} \alpha$ emission line survey of the Andromeda galaxy that overlaps the footprint of the Panchromatic Hubble Andromeda Treasury (PHAT) survey. We found 552 (542) classical Be stars and 8429 (8556) normal B-type stars in epoch \# 1 (epoch \# 2), yielding an overall fractional Be content of $6.15 \% \pm 0.26 \%(5.96 \% \pm 0.25 \%)$. The fractional Be content decreased with spectral subtype from $\sim 23.6 \% \pm 2.0 \%(\sim 23.9 \% \pm 2.0 \%)$ for B0-type stars to $\sim 3.1 \% \pm 0.34 \%(\sim 3.4 \% \pm 0.35 \%)$ for B8-type stars in epoch \# 1 (epoch \# 2). We observed a clear population of cluster Be stars at early fractional main-sequence lifetimes, indicating that a subset of Be stars emerge onto the zero-age main sequence as rapid rotators. Be stars are $2.8 \times$ rarer in M31 for the earliest subtypes compared to the Small Magellanic Cloud, confirming that the fractional Be content decreases in significantly more metal-rich environments (like the Milky Way and M31). However, M31 does not follow a clear trend of Be fraction decreasing with metallicity compared to the Milky Way, which may reflect that the Be phenomenon is enhanced with evolutionary age. The rate of disk-loss or disk-regeneration episodes we observed, $22 \% \pm 2 \% \mathrm{yr}^{-1}$, is similar to that observed for seven other Galactic clusters reported in the literature, assuming these latter transient fractions scale by a linear rate. The similar number of disk-loss events (57) as disk-renewal events (43) was unexpected since disk dissipation timescales can be $\sim 2 \times$ the typical timescales for disk build-up phases.
\end{abstract}

Unified Astronomy Thesaurus concepts: Circumstellar disks (235); Be stars (142); Early-type stars (430); H alpha photometry (691)

Supporting material: machine-readable table

\section{Introduction}

Classical B-type emission line $(\mathrm{Be})$ stars are nonsupergiant stars whose spectra exhibit or have exhibited $\mathrm{H} \alpha$ emission lines (Jaschek et al. 1981), interpreted as arising from an optically thin gaseous circumstellar disk (Struve 1931). A defining property of classical Be stars is their rapid rotation rate, which is estimated to be upwards of 75 percent of the critical rotation rate (Porter \& Rivinius 2003; Rivinius et al. 2013). The velocity structure of gas in these disks is consistent with Keplerian motion (Hummel \& Vrancken 2000; Meilland et al. 2007; Wheelwright et al. 2012) and their behavior is well explained by the viscous decretion disk paradigm of Lee et al. (1991).

A long-standing challenge in the study of classical Be stars has been identifying the mechanism(s) responsible for launching material from the stellar photosphere, while remaining consistent with both the observed velocity structure and the ability of systems to switch between periods in which they exhibit a disk and quiescent periods where all evidence for the presence of a disk disappears. As summarized in Rivinius et al. (2013), nonradial pulsations have been observed for a growing number of Be stars (e.g., Neiner et al. 2002; Rivinius et al. 2003; Baade et al. 2016; Labadie-Bartz et al. 2017), and could be one mechanism that contributes to the ejection of material into Be disks. Baade et al. $(2016,2018)$ have suggested that the so-called "Stefl" frequency of variations might trace star-todisk mass transfer. Internal gravitational wave pulsation modes might also help transport angular momentum from the core to stellar surface (Rogers et al. 2013); Neiner et al. (2012) has shown that stochastically driven g-modes might operate in a subset of Be stars, in addition to the more commonly observed Kappa driven $\mathrm{p}$ - and g-modes. The role of magnetic fields has been considered (Cassinelli et al. 2002; Ud-Doula et al. 2008) but observational studies have failed to find evidence of largescale fields in classical Be stars, despite their observed presence around non-Be B-type stars (Wade et al. 2014). Recent theoretical magnetohydrodynamical simulations of Be disks have corroborated this by showing that field strengths of only $10 \mathrm{G}$ can disrupt Keplerian circumstellar disks (ud-Doula et al. 2018).

Modern stellar evolution models that account for the role of rotation have demonstrated how the metallicity of stars can affect the internal evolution of their angular momentum on the main sequence (Maeder \& Meynet 2000, 2001). It is therefore perhaps not surprising that observations of massive stars in the Galaxy, Large Magellanic Cloud (LMC), and Small Magellanic Cloud (SMC) have shown that the prevalence of classical Be star-disk systems, which are characterized by their rapid 
rotation, increases with decreasing metallicity (Grebel et al. 1992; Maeder et al. 1999; McSwain \& Gies 2005b; Martayan et al. 2006, 2010; Wisniewski \& Bjorkman 2006; Iqbal \& Keller 2013). If the fractional increase in the presence of $\mathrm{Be}$ stars with decreasing metallicity is indeed caused by stars in low metallicity environments having higher stellar rotational velocities (Maeder \& Meynet 2000, 2001; Martayan et al. $2007 b$ ), then one would predict that the prevalence of the Be phenomenon in M31, which is on average $1.5 \times$ solar metallicity (Clayton et al. 2015), would be even less common than in our Galaxy.

Numerous observational and theoretical studies have explored the role that evolutionary age plays in the $\mathrm{Be}$ phenomenon. Stellar evolution models that incorporate the effects of rotation predict that mass loss can dramatically reduce $\Omega / \Omega_{\text {crit }}$ throughout the main-sequence evolution of $\mathrm{O}$ and early B-type stars in Galactic-metallicity environments (Meynet \& Maeder 2000), but $\Omega / \Omega_{\text {crit }}$ can increase, especially near the terminal-age main sequence, in lower metallicity environments like the SMC (Maeder \& Meynet 2001). Given the critical role of rapid rotation in Be disk formation, theory thus suggests that in addition to metallicity, evolutionary age could also play an important role in Be disk formation.

Observationally, it is clear that at least some classical Be stars must emerge on the zero-age main sequence (ZAMS) at near critical rotation rates (e.g., Wisniewski et al. 2007). Moreover, other studies have suggested that disk formation preferentially happens in the second half of a star's mainsequence lifetime (Fabregat \& Torrejón 2000; Martayan et al. 2010), due to internal evolution of a star's angular momentum (Meynet \& Maeder 2000; Maeder \& Meynet 2001) and/or spin-up by companions (McSwain \& Gies 2005b). Further observations that trace the frequency of the Be phenomenon with main-sequence lifetime, stellar mass, and metallicity are needed to discern what drives changes in the internal rotational behavior of B-type stars and whether the effects of metallicity and age can be disentangled.

In this paper, we present the first deep, wide-area, spacebased $\mathrm{H} \alpha$ emission line survey of a portion of M31 that overlaps with the Hubble Space Telescope (HST)-Panchromatic Hubble Andromeda Treasury (PHAT) survey. We focus our analysis on the classical Be star population in M31. We describe the acquisition and reduction of the data in Section 2. We outline our method of identifying classical Be stars in our sample and present the fractional Be content of our sample as a function of spectral type, and detail differences observed in our Be disk population between our two epochs of observations in Section 3. We then discuss the implications of the multiepoch behavior of our data, the dependence of the Be phenomenon on age and metallicity, and the frequency of the Be phenomenon with spectral type in Section 4. Our conclusions are presented in Section 5.

\section{Observations and Data Reduction}

\subsection{Observational Design}

We observed M31 with the HST in the 12 orbit GO-13857 program (Dalcanton PI). Pure-parallel observations were made with the Advanced Camera for Surveys (ACS)/WFC and WFC3/ UVIS camera at three distinct spatial locations that coincided with regions of the galaxy previously observed via the HST/PHAT survey (Dalcanton et al. 2012), yielding six distinct pointings

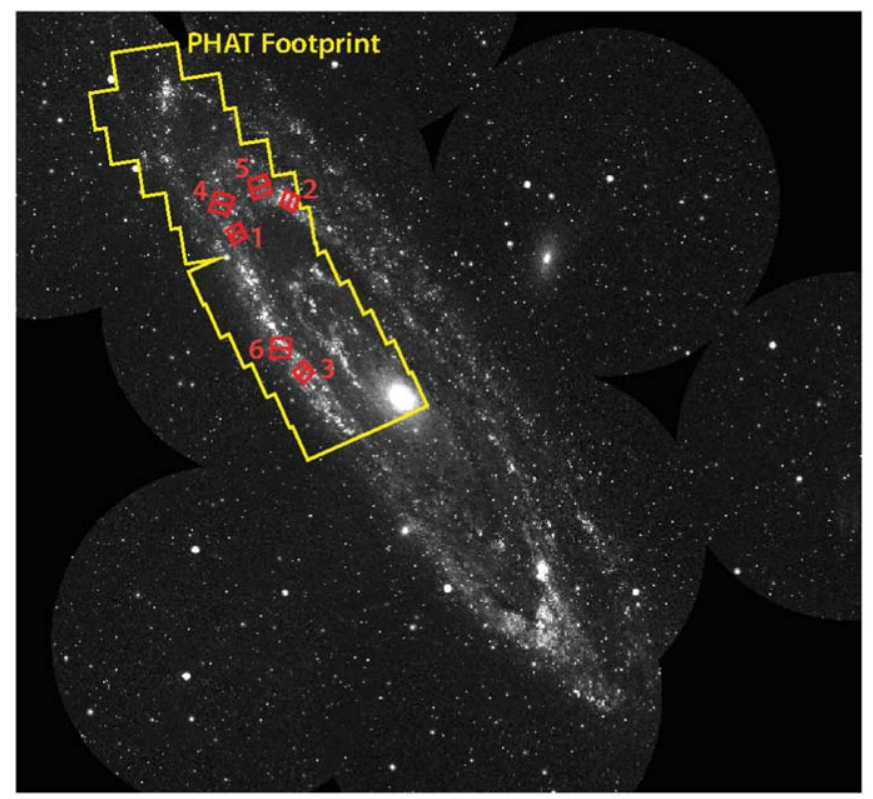

Figure 1. The footprint of our HST emission line survey pointings of M31 are depicted in red and labeled by pointing numbers compiled in Table 1. Also shown here in yellow is the overlay of the full PHAT survey (Dalcanton et al. 2012)

across M31 (Figure 1). We obtained a second epoch of observations of these same six pointings approximately $1 \mathrm{yr}$ later, when HST was able to achieve the same view of these pureparallel pointings. Observations were made in both cameras using the F658N narrow-band filters and using the F625W broadband filter. A summary of our basic observational design is provided in Table 1. As discussed and explained in Section 3.3, although we present the emission line sample derived from both the ACS/ WFC and WFC3/UVIS data, we restricted our Be star statistical analysis to the ACS/WFC data to avoid biasing our results due to the slightly different central wavelengths of the $\mathrm{F} 658 \mathrm{~N}$ filter between the two cameras.

\subsection{Data Reduction}

We utilized the same methodology and pipeline for extracting photometry from our data as used in the PHAT survey, as described in Dalcanton et al. (2012) and Williams et al. (2014). In brief, the images from each epoch of observing (2014 and 2015) for each of the six fields were separately processed through our cloud-based photometry pipeline (Williams et al. 2018), which uses the DOLPHOT photometry package (Dolphin 2000) to force a fit of the precomputed point-spread function on every resolved star in the image stack of greater than $2.5 \sigma$ significance above the expected background level. The photometry from these stars was then aligned and matched to the full 117 million source catalog from the full PHAT survey as presented in Williams et al. (2014) to allow us to compare the stars' narrow-band properties to their six band spectral energy distributions (SEDs). Overall, the photometric reductions were of comparable quality to those of the PHAT survey, except that we found a systematic uncertainty between epochs. Our analysis showed that our broadband exposures covered a short enough portion of the orbit that the thermal expansion and contraction of the optics caused a significant offset between the orbit-averaged point-spread function (PSF) used by DOLPHOT and that of the 
Table 1

Summary of HST Observations

\begin{tabular}{|c|c|c|c|c|c|c|}
\hline $\begin{array}{l}\text { Epoch \# } \\
\ldots\end{array}$ & $\begin{array}{c}\text { Pointing } \\
\ldots\end{array}$ & $\begin{array}{c}\text { Date of Obs } \\
\ldots\end{array}$ & $\begin{array}{c}\text { Camera } \\
\ldots\end{array}$ & $\begin{array}{c}\text { Coordinates } \\
\ldots\end{array}$ & $\begin{array}{c}\text { F625W Expos Time } \\
\text { (s) }\end{array}$ & $\begin{array}{c}\text { F658N Expos Time } \\
\text { (s) }\end{array}$ \\
\hline 1 & 1 & 2014 Oct 28 & WFC3 & $00: 45: 23.38,+41: 46: 12.5$ & 750 & 3850 \\
\hline 2 & 1 & 2015 Oct 5 & WFC3 & $00: 45: 23.38,+41: 46: 12.5$ & 750 & 3850 \\
\hline 1 & 2 & 2014 Oct 1 & WFC3 & $00: 44: 31.02,+41: 52: 08.5$ & 750 & 3850 \\
\hline 2 & 2 & 2015 Aug 29 & WFC3 & $00: 44: 31.02,+41: 52: 08.5$ & 750 & 3850 \\
\hline 1 & 3 & 2014 Sep 30 & WFC3 & $00: 44: 18.05,+41: 20: 53.7$ & 750 & 3850 \\
\hline 2 & 3 & 2015 Sep 25 & WFC3 & $00: 44: 18.05,+41: 20: 53.7$ & 750 & 3850 \\
\hline 1 & 4 & 2014 Oct 28 & $\mathrm{ACS} / \mathrm{WFC}$ & $00: 45: 38.48,+41: 51: 27.3$ & 750 & 3850 \\
\hline 2 & 4 & 2015 Oct 5 & ACS/WFC & $00: 45: 38.49,+41: 51: 27.3$ & 750 & 3850 \\
\hline 1 & 5 & 2014 Oct 1 & $\mathrm{ACS} / \mathrm{WFC}$ & $00: 45: 00.29,+41: 54: 32.6$ & 750 & 3850 \\
\hline 2 & 5 & 2015 Aug 29 & $\mathrm{ACS} / \mathrm{WFC}$ & $00: 45: 00.29,+41: 54: 32.6$ & 750 & 3850 \\
\hline 1 & 6 & 2014 Sep 30 & ACS/WFC & $00: 44: 40.57,+41: 25: 05.3$ & 750 & 3850 \\
\hline 2 & 6 & 2015 Sep 25 & ACS/WFC & $00: 44: 40.57,+41: 25: 05.3$ & 750 & 3850 \\
\hline
\end{tabular}

Note. Summary of HST observations of M31 from GO-13857.

F625W images across epochs, which introduced a systematic error that we further discuss in Appendix.

Next, our source catalog was enhanced by adding the output of the Bayesian Extinction And Stellar Tool (BEAST; Gordon et al. 2016) on the PHAT catalog data, which added $T_{\text {eff }}, \log g$, and age information for each source. Gordon et al. (2016) developed the BEAST to model the observed broad SED of an individual star by considering the full observational uncertainties. The BEAST primarily returns the posterior probability distributions for the intrinsic stellar properties of individual stars (e.g., stellar temperature, age, surface gravity) and their line-of-sight dust information (dust column density, grain size distribution, composition). The BEAST has been successfully used for many multiband $H S T$ programs such as PHAT (PI: Dalcanton), SMIDGE (PI: Sandstrom), METAL (PI: RomanDuval), and the Local Volume UV survey (PI: Gilbert). Specifically, Gordon et al. (2016) ran the BEAST for all PHAT stars that were detected in $\mathrm{F} 475 \mathrm{~W}$ and at least three more bands (among F225W, F336W, F814W, F110W, and F160W) with resolutions of 0.15 in log age, 0.15 in $\mathrm{Av}, 0.25$ in $\mathrm{fA}$, and $Z=0.004,0.008,0.019$, and 0.03. For this study, we crossmatched this PHAT-BEAST catalog with our classical Be star candidates, and used the BEAST measurements to better categorize and understand Be stars in M31. Finally, we corrected our data for focus-induced offsets, as described in Appendix.

\section{Analysis}

\subsection{Defining the Sample}

The initial reduced data set contained approximately two million stars in each epoch (Figure 2). As this paper focuses solely on the analysis of the classical Be population of the survey, we applied several criteria to isolate the population of main-sequence B-type stars with high quality data from the larger survey database. Our basic process was to utilize the value-added nature of BEAST-derived fundamental stellar parameters to isolate the population of main-sequence B-type stars in our sample, and then use traditional color-magnitude diagrams (CMDs) to confirm the robustness of these inferred stellar parameters.

We began our sample definition by requiring sources in the new observations to have a signal-to-noise ratio $(\mathrm{S} / \mathrm{N})$ of $>10$

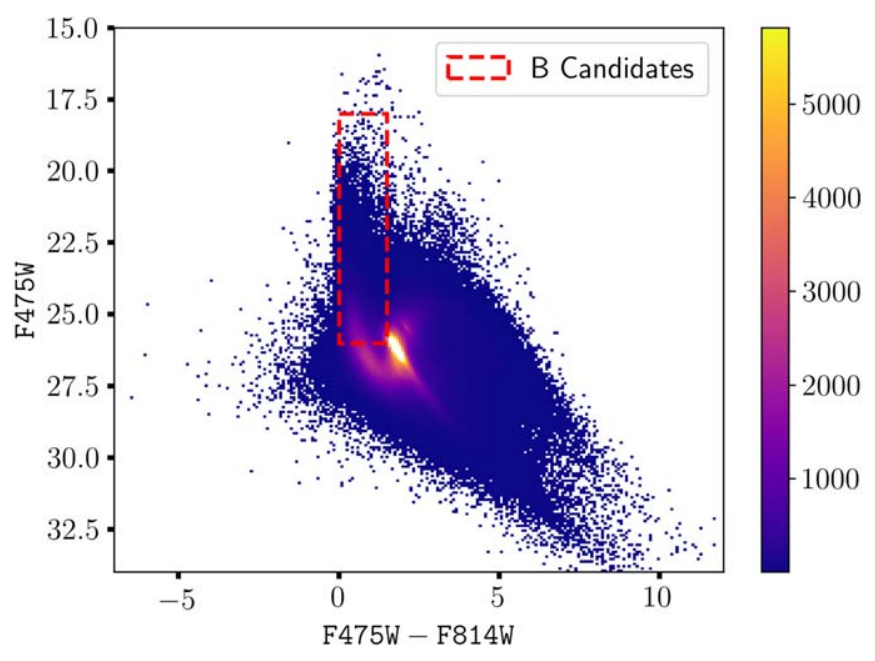

Figure 2. A color-magnitude diagram of all $\sim 2$ million point sources detected via our program, without excluding any sources based on their intrinsic properties or observed errors.

in the F475W, F625W, F658N, and F814W filters, which are the primary filters that we used in our subsequent analysis. Next, we required sources have BEAST-derived 50th percentile effective temperatures that resided within the range expected for B-type stars, $4.00<\log \left(T_{\text {eff }}\right)<4.51$ (Underhill et al. 1979). We also required that sources have a BEAST-derived 50th percentile surface gravity, inclusive of $+1 \sigma_{\text {err }}$ computed from the 84th percentile surface gravity, of $\log (g)>3.75$, to isolate main-sequence stars and exclude giants and supergiants (Castelli \& Kurucz 2004).

We inspected the distribution of these selected B-star candidates on CMDs to determine whether uncertainties associated with BEAST-derived stellar parameters were introducing large populations of likely false-positive B-type main-sequence stars. We found that adopting a more conservative surface gravity criteria $\left(\log (g)_{16}>3.75\right.$, where $\log (g)_{16}$ is the 16 th percentile of the $\log (g)$ posterior probability distribution) led to a $4 \times$ reduction in the number of sources, but these extra sources that were removed occupied the same region on the $\mathrm{CMD}$ as our higher confidence main-sequence B stars. As such, we adopted our less conservative criterion $\left(\log (g)_{84}>3.75\right)$, which had the net effect of being most effective at excluding only the supergiants from our 


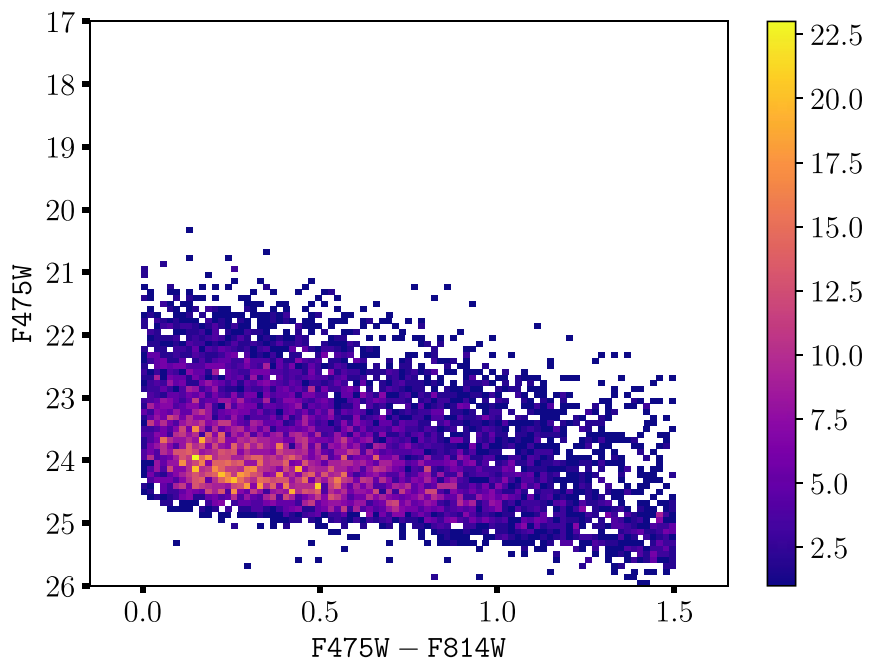

Figure 3. A color-magnitude diagram of all point sources that remained after the application of the data quality criteria.

sample while retaining the main-sequence stars. Based on these inspections, we included additional criteria to reject sources having $(\mathrm{F} 475 \mathrm{~W}-\mathrm{F} 814 \mathrm{~W})<0$, which were likely planetary nebulae (see, e.g., Veyette et al. 2014), and also rejected objects having $(\mathrm{F} 475 \mathrm{~W}-\mathrm{F} 814 \mathrm{~W})>1.5$, as these sources had BEASTderived $\log (g)$ values near 3.75 and were clearly isolated from the bulk population with $\operatorname{larger} \log (g)$ values, indicating they were likely post-main-sequence giants. Finally, we excluded a small number of sources in our catalog that were assigned the same R.A./decl. coordinates by our matching routine between filters, due to source confusion between different survey tiles. The final data set for pointings 4-6 matching the aforementioned criteria contained 8981 stars in epoch one and 9098 in epoch two, and their CMD positions are plotted in Figure 3.

\subsection{Identifying Be Stars}

Classical Be stars by definition are stars that exhibit or have exhibited $\mathrm{H} \alpha$ emission, which is interpreted as originating from their circumstellar gas disks (Jaschek et al. 1981). As such, one technique commonly used to identify classical Be stars is the use of photometric two-color diagrams (2-CDs) that utilize a narrow-band filter centered on $\mathrm{H} \alpha$ and an associated filter that samples the nearby continuum region (see, e.g., Grebel et al. 1992; Keller et al. 1999; McSwain \& Gies 2005a, 2005b; Wisniewski \& Bjorkman 2006). We discuss some of the limitations of this diagnostic in more detail in Section 3.2.1. We adopted a similar diagnostic approach here, using new data in the F625W and F658N HST filters from our GO-13857 program to diagnose the presence of emission or absorption in $\mathrm{H} \alpha$, along with archival data in the F475W and F814W HST filters from the PHAT survey (Williams et al. 2014).

We first computed synthetic photometry through the known properties of HST's optics and cameras for normal B-type main-sequence stars spanning a range of $B$ subtypes in the F625W and F658N filters. We began by using pysynphot (STScI Development Team 2013) and the Castelli-Kurucz Atlas (Castelli \& Kurucz 2004) to create synthetic stellar spectra for five subtypes, B0, B1, B3, B5, and B8, adopting the stellar parameters listed in Table 2, based on average values for each subtype compiled in Underhill et al. (1979). These spectra were created using pysynphot's Icat function, which
Table 2

Synthetic Spectra Properties

\begin{tabular}{lcccc}
\hline \hline $\begin{array}{l}\text { Spectral } \\
\text { Type }\end{array}$ & Temperature & $\log ($ Gravity) & Log(Metallicity) & $\begin{array}{c}\text { H } \alpha \\
\text { EW (A) }\end{array}$ \\
\hline B0V & 30000 & 3.90 & -1.7 & 2.83 \\
B1V & 25400 & 3.90 & -1.7 & 3.52 \\
B3V & 18700 & 3.94 & -1.7 & 4.67 \\
B5V & 15400 & 4.04 & -1.7 & 5.71 \\
B8V & 11900 & 4.04 & -1.7 & 7.52 \\
\hline
\end{tabular}

Note. Synthetic spectra parameters were obtained from the recommended values in the Castelli-Kurucz Atlas; the metallicity adopted was $1.5 \times$ solar, as taken from Clayton et al. (2015).

interpolates between the discrete models in the Castelli-Kurucz Atlas. We adopted a color excess of $E(B-V)=0.4$, appropriate for moderately reddened stars in M31 (Clayton et al. 2015), along with $R_{v}=3.1$. This adopted color excess is consistent with the average $E(B-V)$ values computed for our sample of M31 B-type stars via the BEAST (Gordon et al. 2016). We note that the adoption of a color excess appropriate for moderately reddened stars will lead to a more stringent color criterion for the identification of Be stars in our data, such that our Be star rates will be a lower limit. We used the Extinction function to apply reddening to our spectra, yielding photospheric $\mathrm{H} \alpha$ absorption line profiles that are representative of main-sequence B-type stars (or classical Be stars when they are in a "diskless" state). We compile the equivalent widths of these $\mathrm{H} \alpha$ absorption lines in Table 2.

The amount of $\mathrm{H} \alpha$ emission that a classical Be star exhibits is to first order representative of the amount of disk material present within the stellocentric distances probed by $\mathrm{H} \alpha$ (Rivinius et al. 2013) at any epoch. We next used the absorption spectra we created in the previous step to create emission line spectra representative of a classical Be star with an arbitrarily strong disk (e.g., having $\mathrm{H} \alpha$ EWs as compiled in Table 2, albeit in emission), and spectra whose photospheric absorption is merely filled in with emission, representative of a classical Be star with a more tenuous disk (e.g., having $\mathrm{H} \alpha$ EWs of 0 ). We fit a Guassian to the $\mathrm{H} \alpha$ line using curve_fit from scipy.optimize, and then used this expression to create profiles with filled-in photospheric absorption and pure emission signatures. Finally, we computed the synthetic magnitudes in the F625W and F658N filters for the ACS and WFC3 cameras for these spectra for each B subtype to allow us to deduce which of the B-type stars in our sample were plausibly classical Be stars.

Our identification of classical Be stars was made using twocolor diagrams (2-CDs) developed for each of our spectral subtypes (e.g., Figures 4-8) set by the 50th percentile $T_{\text {eff }}$ reported by the BEAST. The boundaries of different spectral subtype assignments are simply the midpoint $T_{\text {eff }}$ between subtypes listed in Table 2. Because the $T_{\text {eff }}$ values were inferred from broadband photometric observations by the BEAST, we expect our spectroscopic subtype assignments could differ by 1-2 subtypes. However, because we binned the spectroscopic classifications in Section 3.3, minor misclassifications do not impact our overall results. We also explored effects of including $1 \sigma_{\text {err }}$ in our 50th percentile $T_{\text {eff }}$ source catalog cuts, and found that this only changed our final fractional Be ratios $\pm 1 \%$.

The dotted lines in each 2-CD demarcate the expected values for pure photospheric absorption (black), filled-in absorption 


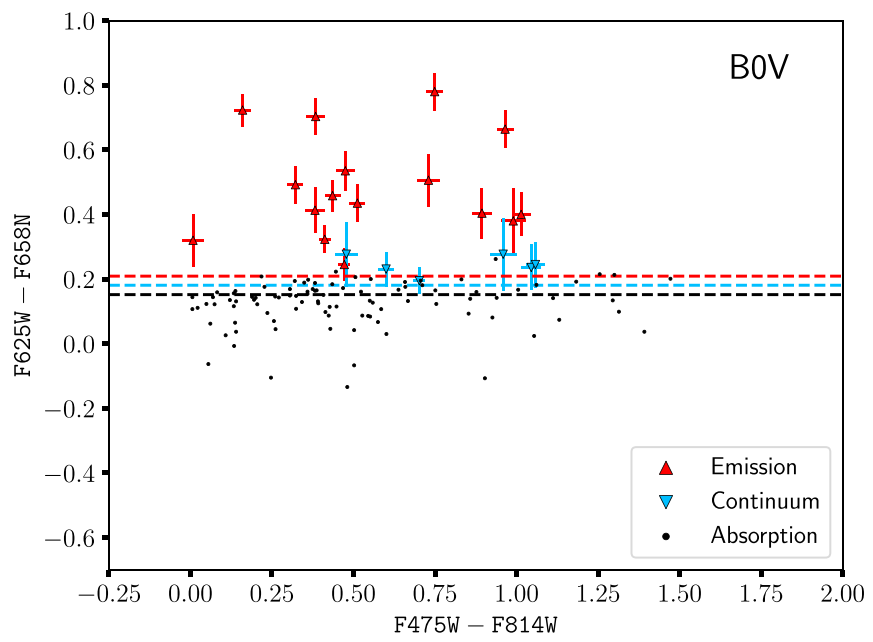

Figure 4. A representative 2-CD for our B0V spectral type sources, from epoch 1 , pointing 5 . We classified objects as classical Be stars if their $(\mathrm{F} 625 \mathrm{~W}$ $-\mathrm{F} 658 \mathrm{~N})$ colors, including $3 \sigma$ errors, are redder than the photospheric absorption level. The horizontal dashed lines in these figures depict the colors expected for pure $\mathrm{H} \alpha$ photospheric absorption (black), filled-in absorption (blue), and pure $\mathrm{H} \alpha$ emission profiles (red) as computed from our models. Note that the plotted error bars are $3 \sigma$ errors.

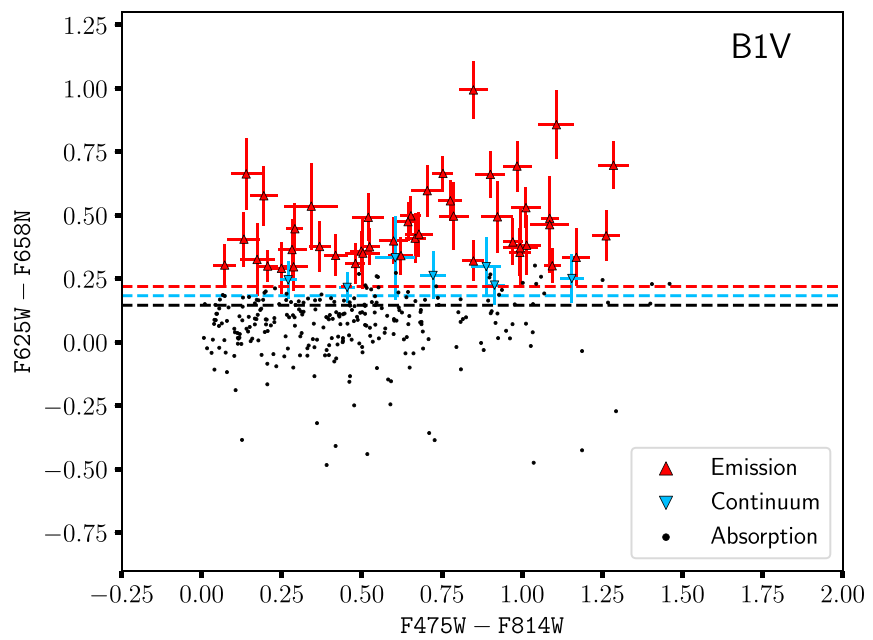

Figure 5. A representative 2-CD for our B1V spectral type sources, from 1 epoch of a pointing. The description of the data presentation is the same as in Figure 4.

(blue), and pure emission (red) profiles from our synthetic spectra. We adopted conservative limits for identifying sources as classical Be stars, requiring their $(\mathrm{F} 625 \mathrm{~W}-\mathrm{F} 658 \mathrm{~N})$ colors to be redder than the photospheric absorption level (e.g., [(F625W-F658N) $\left.-3 \sqrt{\left(\mathrm{F} 625 \mathrm{~W}_{\mathrm{err}}\right)^{2}+\left(\mathrm{F} 658 \mathrm{~N}_{\mathrm{err}}\right)^{2}}\right]>$ photospheric levels). Stated differently, we conservatively defined normal B-type stars without circumstellar disks as either (1) being located below the dotted black line or (2) including the black line within their $3 \sigma$ error interval in Figures 4-8. Such normal B-type stars are depicted by black circles in these figures.

We further broke down the Be population into two categories: stars whose $3 \sigma$ error interval extended blueward of the pure emission cutoff (potentially weaker disk systems, depicted by blue triangles) and stars whose $3 \sigma$ error interval denoted it unambiguously as a strong disk system (depicted by red triangles). We were unable to identify any correlations between the amplitude of (F625W-F658N) excess and (F475-F814W)

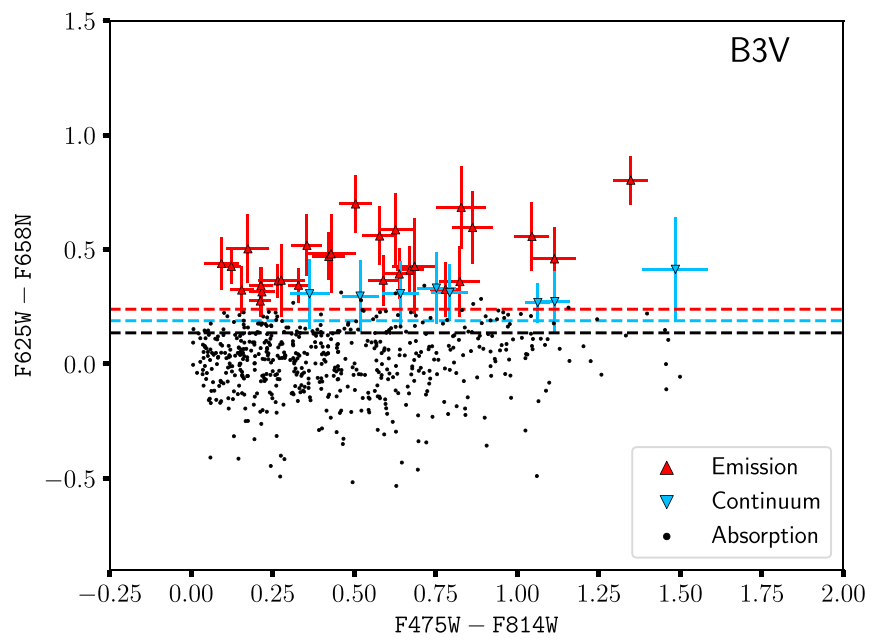

Figure 6. A representative 2-CD for our $\mathrm{B} 3 \mathrm{~V}$ spectral type sources, from 1 epoch of a pointing. The description of the data presentation is the same as in Figure 4.

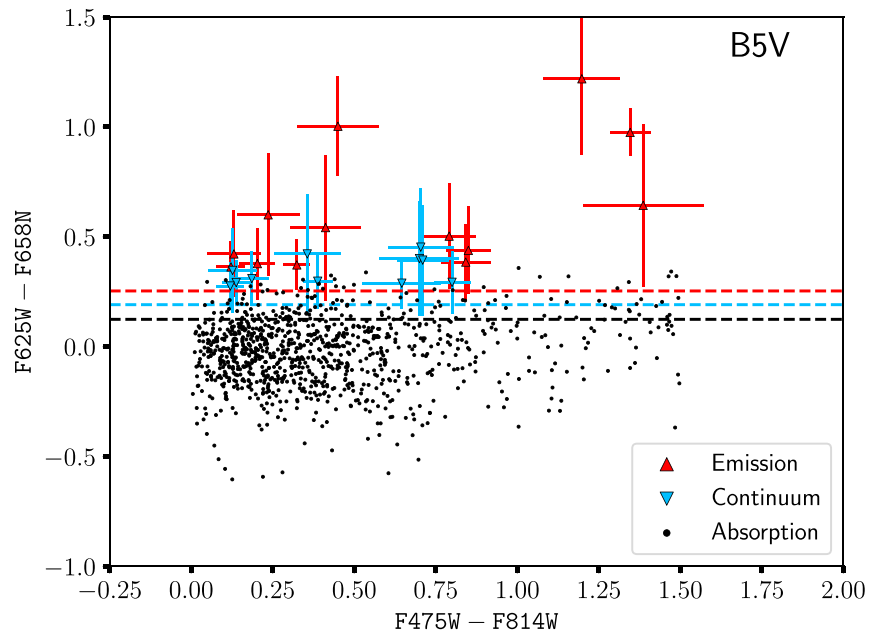

Figure 7. A representative 2-CD for our B5V spectral type sources, from 1 epoch of a pointing. The description of the data presentation is the same as in Figure 4.

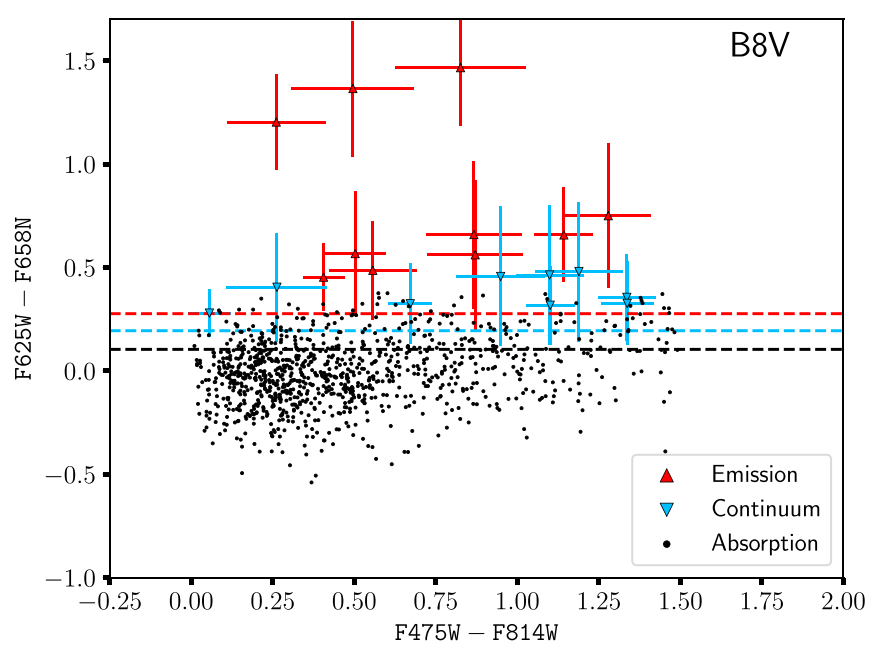

Figure 8. A representative 2-CD for our B8V spectral type sources, from 1 epoch of a pointing. The description of the data presentation is the same as in Figure 4. 
colors, indicating that the presence of red optical continuum excesses (Rivinius et al. 2013) did not bias us against detecting the strongest disk systems based on our selection criteria.

\subsubsection{Limitations of the 2-CD Technique}

It is well established that 2-CDs can identify other astrophysical sources that emit at $\mathrm{H} \alpha$ besides classical $\mathrm{Be}$ stars, such as supergiants, $\mathrm{B}[e]$ stars, and Herbig Be stars. The level of this contamination is expected to be lower when the diagnostic is used in cluster populations (see, e.g., Wisniewski \& Bjorkman 2006 and references therein). Moreover, for our survey, as discussed in Section 3.1, we were able to use the value-added characterization of the fundamental stellar properties of our data set via the BEAST to exclude sources that had surface gravities that indicated they were not main-sequence stars. As such, our 2-CDs should be cleaner from contaminants than other purely photometric investigations.

It is also prudent to consider the limitations of our 2-CD diagnostic given the wealth of $\mathrm{H} \alpha$ emission line profile morphologies and variability that classical Be stars have been shown to exhibit. We explored these limitations both using published literature and compiled spectra of Be stars from the Be Star Spectra (BeSS) database (Neiner et al. 2011). The ACS/WFC F $658 \mathrm{~N}$ filter has a central wavelength of $6584 \AA$, a FWHM of $87.5 \AA$, and $>1 \%$ throughput across $\sim 130 \AA$ (ACS Instrument Handbook v19.0). As such, this filter is sufficiently broad to capture the full range of $\mathrm{H} \alpha$ line profile morphologies for each spectral subtype of classical Be star.

One can readily compare our $(\mathrm{F} 625 \mathrm{~W}-\mathrm{F} 658 \mathrm{~N})$ color selection criteria for $\mathrm{Be}$ stars against the range of $\mathrm{H} \alpha$ emission line strengths that classical Be stars exhibit, as this criteria is based on the photospheric absorption EWs of each B subtype (Table 2). To aid in this process, we show reference ( $\mathrm{F} 625 \mathrm{~W}-$ $\mathrm{F} 658 \mathrm{~N})$ colors for filled-in absorption $(\mathrm{H} \alpha \mathrm{EW}=0)$ and moderate emission strengths $\left(\mathrm{EW}_{\text {emission }}|=| \mathrm{EW}_{\text {absorption }} \mid\right)$ in Figures 4-8. We have confirmed that the $\mathrm{H} \alpha \mathrm{EW}$ of emission line profiles from moderately sized stable and stochasically variable Be disks are typically well in excess of the red dashed line in Figures 4-8 (e.g., they have emission EWs well in excess of the underlying photospheric absorption line; Rivinius et al. 1998; Neiner et al. 2011; Draper et al. 2014). Our selection criteria, for example, would recover $\sim 87 \%$ of the 76 star sample of Be stars spanning a range of spectral subtypes, based on single to few epoch $\mathrm{H} \alpha$ EWs presented in Slettebak et al. (1992), Mennickent et al. (1994), and Silaj (2014). We confirmed this is also true for many Be shell stars (Rivinius et al. 2006), despite the fact that their $\mathrm{H} \alpha$ profiles are depressed by narrow central absorption components (Neiner et al. 2011; Draper et al. 2014). An exception is the subset of shell stars with particularly weak disks, where the net $\mathrm{H} \alpha$ EW becomes dominated by the underlying photospheric absorption line. Even classical Be stars experiencing an active disk dissipation episode often retain $\mathrm{H} \alpha$ EWs above our detection criteria, until the end stages of their disk loss (see, e.g., the cases of 60 Cyg and $\pi$ Aqr, Wisniewski et al. 2010). By contrast, the early stages of disk rebuilding episodes as well as the weakest Be disks are often characterized by $\mathrm{H} \alpha$ EWs at or below filled-in photospheric absorption levels $(\mathrm{EW}=0$; dashed blue line in Figures 4-8; e.g.,Wisniewski et al. 2010; Grundstrom et al. 2011; Neiner et al. 2011), and so would likely not be detected by our selection criteria.
Table 3

Summary of \# Be/(\# Be + \# Normal B) Stars

\begin{tabular}{lcc}
\hline \hline Pointing & Epoch 1 Be Fraction $\pm 1 \sigma$ & Epoch 2 Be Fraction $\pm 1 \sigma$ \\
\hline 4 & $5.39 \% \pm 0.42 \%$ & $5.69 \% \pm 0.43 \%$ \\
5 & $5.25 \% \pm 0.40 \%$ & $5.45 \% \pm 0.40 \%$ \\
6 & $7.76 \% \pm 0.48 \%$ & $6.76 \% \pm 0.46 \%$ \\
Overall & $6.15 \% \pm 0.26 \%$ & $5.96 \% \pm 0.25 \%$
\end{tabular}

Note. This table summarizes the fraction of Be stars (\# Be/(\# Be + \# normal B)) stars detected in each epoch for pointings 4-6, along with $\sigma$ uncertainties. Note that pointings $1-3$ are excluded from the overall fractions compiled in this table.

Table 4

Summary of \# Be/(\# Be + \# Normal B) Stars as a Function of Spectral Type

\begin{tabular}{lcccc}
\hline \hline Epoch & $\begin{array}{c}\text { Spectral } \\
\text { Type }\end{array}$ & $\begin{array}{c}\text { \# Be } \\
\text { Stars }\end{array}$ & $\begin{array}{c}\text { (\# Normal B }+\# \\
\text { Be Stars) }\end{array}$ & Be Fraction $\pm 1 \sigma$ \\
\hline 1 & B0V & 106 & 450 & $23.56 \% \pm 2.00 \%$ \\
1 & B1V & 163 & 1148 & $14.20 \% \pm 1.03 \%$ \\
1 & B3V & 112 & 1981 & $5.65 \% \pm 0.52 \%$ \\
1 & B5V & 91 & 2840 & $3.20 \% \pm 0.33 \%$ \\
1 & B8V & 80 & 2562 & $3.12 \% \pm 0.34 \%$ \\
1 & Overall & 552 & 8981 & $6.15 \% \pm 0.26 \%$ \\
2 & B0V & 107 & 448 & $23.88 \% \pm 2.01 \%$ \\
2 & B1V & 159 & 1152 & $13.80 \% \pm 1.01 \%$ \\
2 & B3V & 119 & 1965 & $6.06 \% \pm 0.54 \%$ \\
2 & B5V & 68 & 2895 & $2.35 \% \pm 0.28 \%$ \\
2 & B8V & 89 & 2638 & $3.37 \% \pm 0.35 \%$ \\
2 & Overall & 542 & 9098 & $5.96 \% \pm 0.25 \%$ \\
\hline
\end{tabular}

Note. Be Star fractions for epochs 1 and 2, using pointings 4-6 combined.

\subsection{M31's Total Be Star Population}

Our methodology identified 552 Be stars out of 8981 (B + Be) stars in epoch 1, and $542 \mathrm{Be}$ stars out of $9098(\mathrm{~B}+\mathrm{Be})$ stars in epoch 2. The resultant $\mathrm{Be}$ fraction (\# Be/(\# normal B $+\# \mathrm{Be})$ ) for each pointing is shown in Table 3 , and is broken down as a function of spectral subtype in Table 4 . We note that pointings 1-3, obtained from the WFC3 camera (see Section 2.1), are excluded from these statistical analyses for the remainder of the paper, as the filter utilized $(\mathrm{F} 658 \mathrm{~N})$ only sampled the wing of $\mathrm{H} \alpha$, therefore biasing us against robustly detecting weaker disk signals. We provide comprehensive information about each normal B-type star and classical Be star in the catalog in Table 8.

The overall average \# Be/(\# normal $\mathrm{B}+\# \mathrm{Be}$ ) stars (in pointings $4-6$ ) is $\sim 6 \%$, with no statistically significant difference between epoch $1(6.15 \% \pm 0.26 \%)$ and epoch 2 $(5.96 \% \pm 0.25 \%)$. These overall Be fractions are strongly impacted by the larger number of later spectral type sources we detected, as seen in Table 4 and Figure 9. In particular, Figure 9 illustrates the clear trend of the Be fraction as a function of spectral type. The Be fraction is largest for B0-type stars and progressively decreases for later subtypes.

We caution that our detection rates as a function of spectral type in Table 4 and Figure 9 are likely subject to several biases. The total main-sequence B-type star population (i.e., column 4 in Table 4) exhibits incompleteness based on expectations from an initial mass function (IMF). Assuming no incompleteness at the B0 subclass and adopting a Kroupa IMF (Kroupa 2001), our B-type star population is incomplete by a factor of $1.2 \times(\mathrm{B} 1)$, 


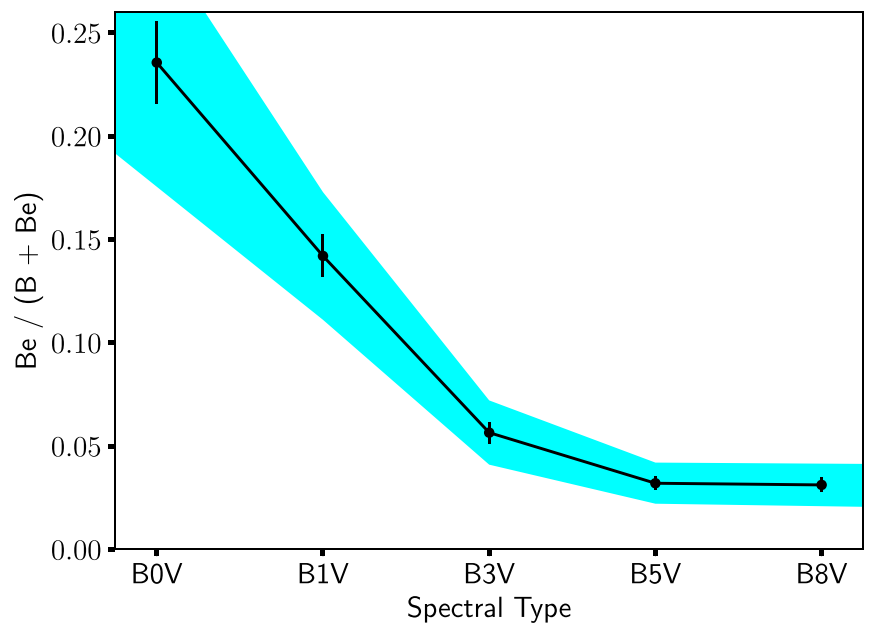

Figure 9. The \# Be/(\# normal B + \# Be) fraction as a function of spectral type, as compiled from Table 4 , is shown. Note that the black error bars represent the $1 \sigma$ interval and the blue shading represents the $3 \sigma$ interval.

$1.6 \times(\mathrm{B} 3), 2.1 \times(\mathrm{B} 5)$, and $6.3 \times(\mathrm{B} 8)$. Of course if this incompleteness affects our recovery of Be stars at the same rate it does normal B-type stars, the fractional Be content we nominally derived in Table 4 and Figure 9 would remain unchanged. We also note that the larger (F625W-F658N) photometric errors present for later spectral subtypes acts as another factor that biases us against detecting weaker disk systems in later spectral subtypes. Since we do not a priori know the intrinsic prevalence of strong versus weaker disk systems as a function of spectral subtype, we do not quantify an upper limit to our quoted Be fractions owing to this systematic.

\subsection{Variability in M31's Be Star Population}

The wide (year-long) time baseline of our two epochs of observations allows us to assess the statistical prevalence of diskloss and disk-renewal episodes, albeit the poor temporal sampling limits our ability to constrain the detailed timescale of these events. Because of the circumstellar disk's H $\alpha$-emitting properties, large changes in the size of these disks will cause sources to significantly move in 2-CDs. We classified a Be star as exhibiting a disk-loss or disk-renewal event if it was determined to be a Be star in one epoch and its (F625W-F658N) color was at or bluer than the expected value for a normal B-type star for the other, and viceversa. We show the evolution of such disk-loss and disk-renewal events in 2-CD space for B0V-type stars in Figure 10. Following McSwain et al. (2009), we defined the percentage, $p$, of transient Be stars, as the ratio of Be stars that exhibit either disk-loss or diskrenewal events, $n_{\text {Betrans }}$, relative to the total number of Be stars, $n_{\text {Betotal: }}$

$$
p=\frac{n_{\text {Betrans }}}{n_{\text {Betotal }}} .
$$

We also defined the error in this percentage to be:

$$
\sigma_{p}=\sqrt{\frac{p(1-p)}{n_{\text {Betotal }}}} .
$$

Approximately $13 \%$ of our sample exhibited a disk-loss event while $\sim 10 \%$ of our sample exhibited a disk-renewal event (Table 5). Roughly $77 \%$ of our sample maintained their status of having a disk at some level through the $1 \mathrm{yr}$ baseline of our data.
As seen in Figure 10 for our B0-type stars, such nontransient systems still exhibited evidence of variability in their $\mathrm{H} \alpha$ emission. Note that the total number of Be stars reported in both epochs in Table 5 is lower than the number reported in epoch \# 1 or \# 2 in Table 4 as some sources had too low $\mathrm{S} / \mathrm{N}$ to meet our source catalog requirements in one of the two epochs.

\subsection{Cluster versus Field Be Stars}

Ascertaining whether the stars in our catalog belong to distinct clusters or a general field population is of interest as the uniform age of cluster members can allow one to assess how the Be phenomenon evolves with a star's fractional mainsequence lifetime. We matched our source catalog against clusters identified by the citizen science-based Andromeda Project and compiled in Johnson et al. (2015). Specifically, we required our sources to be within the quoted aperture radius for each cluster, $\mathrm{R}_{a p}$, as compiled in Johnson et al. (2015).

Of the $8981 \mathrm{~B}+\mathrm{Be}$ stars we detected in epoch 1, we classified 420 cluster stars in 85 unique clusters and 8561 field stars. We computed a corresponding fractional Be content of $16.19 \% \pm 1.80 \%$ in cluster environments and a much smaller fractional Be content of $5.65 \% \pm 0.25 \%$ in field environments (Table 6). Our results from epoch 2 are consistent with those found in epoch 1 to within errors. In epoch 2, of the $9098 \mathrm{~B}+$ Be stars, we classified 417 as cluster stars in 86 unique clusters and 8681 as field stars, and computed a fractional Be content of $14.39 \% \pm 1.72 \%$ in cluster environments and a much smaller fractional Be content of $5.55 \% \pm 0.24 \%$ in field environments (Table 6). Be stars are located in 23 unique clusters in epoch 1 and 27 unique clusters in epoch 2. We caution that our photometry is likely incomplete or of worse quality in the densest cluster environments, as source contamination from blends could be more detrimental.

The distribution of ages for our Be stars located in clusters, adopted from the cluster ages determined and tabulated by Johnson et al. (2016), is shown in Figure 11. We also computed the fractional main-sequence lifetime for each cluster star, defined as the ratio of a star's age to its main-sequence lifetime. This was done by assigning a mass to each inferred spectral subtype, taken from Table 1 of Silaj et al. (2014), and adopting main-sequence lifetimes for solar metallicity stars, assuming $\Omega / \Omega_{\text {crit,initial }}=0.8$, from interpolating Table 2 of Georgy et al. (2013). The resultant distribution of our cluster sample as a function of fractional main-sequence lifetime is shown in Figure 11 . We remark that this methodology initially assigned 12 of our sources with fractional main-sequence lifetimes exceeding 1, likely due to improper main-sequence lifetime assignment related to the 1-2 spectral subtype uncertainty of our sample (Section 3.2), and were reassigned fractional mainsequence lifetimes of 1 . We further discuss and interpret trends in these data in Section 4.2.

\section{Discussion}

\subsection{Variability}

A remarkable aspect of the Be phenomenon is dramatic episodes of complete disk-loss and disk-regeneration. Longterm photometric, spectroscopic, and polarimetric studies have suggested that complete dissipation of some disks occurs over timescales as long as one to several years (e.g., Wisniewski et al. 2010; Carciofi et al. 2012), whereas partial disk-growth episodes imprint a variety of clear observational signatures over 

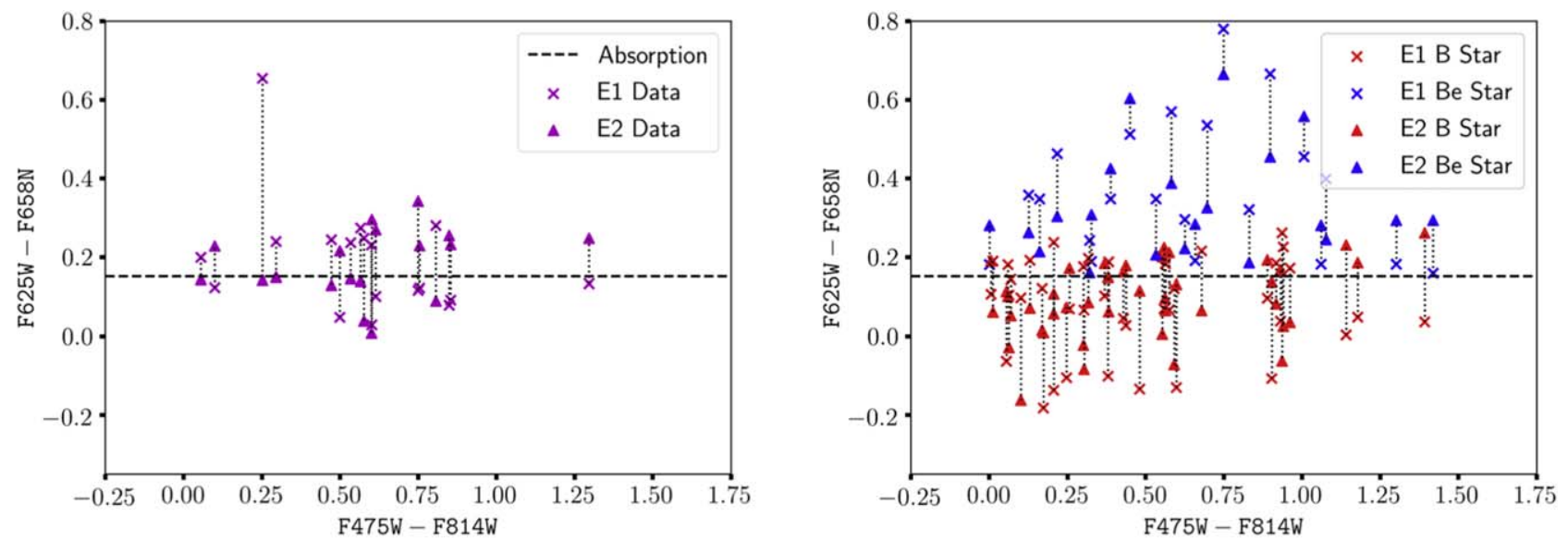

Figure 10. A plot of the variability of B0V stars in our data set. The left-hand panel shows how stars that exhibited disk-loss or disk-renewal events (transient Be stars) evolved between the first and second epoch. The right-hand plot depicts the variability that stable B and stable Be stars exhibited between the two epochs of observations.

Table 5

Disk-loss and Disk-renewal Episodes

\begin{tabular}{|c|c|c|c|c|c|}
\hline Class & $\begin{array}{l}\text { \# Main- } \\
\text { tained Disk }\end{array}$ & $\begin{array}{c}\# \\
\text { Disk- } \\
\text { Loss }\end{array}$ & $\begin{array}{l}\text { \# Disk- } \\
\text { Renewal }\end{array}$ & $\begin{array}{l}\text { otal \# Be } \\
\text { Stars in } \\
\text { Both } \\
\text { Epochs }\end{array}$ & $p$ \\
\hline B0V & 80 & 9 & 9 & 98 & $18 \% \pm 4 \%$ \\
\hline B1V & 121 & 14 & 11 & 146 & $17 \% \pm 3 \%$ \\
\hline B3V & 79 & 12 & 15 & 106 & $25 \% \pm 4 \%$ \\
\hline B5V & 40 & 13 & 6 & 59 & $32 \% \pm 6 \%$ \\
\hline B8V & 32 & 9 & 2 & 43 & $26 \% \pm 7 \%$ \\
\hline Total & 352 & 57 & 43 & 452 & $22 \% \pm 2 \%$ \\
\hline
\end{tabular}

Note. A summary of the number of sources (both cluster and field) that gained or lost a disk through the $1 \mathrm{yr}$ duration of our observations. The percentage of transient systems exhibiting disk-loss or disk-renewal, $p$, is also given.

Table 6

Prevalence of Be Stars in Cluster vs. Field Environments

\begin{tabular}{lcccc}
\hline \hline Epoch & Location & \# Be Stars & \# Normal B Stars & Be Fraction $\pm 1 \sigma$ \\
\hline 1 & Field & 484 & 8077 & $5.65 \% \pm 0.25 \%$ \\
1 & Cluster & 68 & 352 & $16.19 \% \pm 1.80 \%$ \\
2 & Field & 482 & 8199 & $5.55 \% \pm 0.24 \%$ \\
2 & Cluster & 60 & 357 & $14.39 \% \pm 1.72 \%$ \\
\hline
\end{tabular}

Note. The fractional Be content (\# Be/(\# normal B + \# Be)) for objects identified as likely belonging to cluster and field environments is tabulated for both epochs of our observations.

timescales of days to months to years (Rivinius et al. 1998; Grundstrom et al. 2011; Draper et al. 2014; Labadie-Bartz et al. 2017, 2018). Both observational and theoretical studies have suggested that the disk dissipation phase can last for $\sim 2 \times$ longer than the typical timescale for disk build-up phases (Haubois et al. 2012; Vieira et al. 2017; Labadie-Bartz et al. 2018). Since each observational bandpass/diagnostic probes different physical regions within Be disks (Haubois et al. 2012; Rivinius et al. 2013), it is important to test this different disk growth versus dissipation timescale prediction of the viscous decretion disk model (Haubois et al. 2012) with a variety of observational techniques.

These events plausibly represent epochs in which the amplitude or efficacy of the disk driving mechanisms change.
Diagnosing both the timescale and frequency of such events therefore seems likely to elucidate the fundamental mechanism (s) driving the phenomenon. Early studies of the frequency of transient events were limited, based only on small samples of Galactic clusters (e.g., $45 \mathrm{Be}$ stars distributed across seven clusters; McSwain et al. 2008, 2009), revealing a wide dispersion in the observed transient percentage $(0 \% \pm 18 \%$ to $75 \% \pm 22 \%$, with a composite mean of $51 \% \pm 7 \%$; McSwain et al. 2009). Granada et al. (2018) characterized the IR color variability and location in IR CMD and 2-CD space of a sample of Be stars in open clusters to infer that $\sim 9 \%-15 \%$ of their sample of Be stars had a dissipating or small disk. Larger volume surveys have quantified that long-term variations (LTVs) in the $R$-band occur over many years in $37 \%(81 /$ 217) of Be stars (Labadie-Bartz et al. 2017). However, LTVs are not classified on the strict basis of complete dissipation of an observational signature of a disk or the regeneration of a disk from a diskless state, and include partial dissipation and build-up events.

Our survey provides a significant statistical boost to previous studies of the frequency of transient events, i.e., a $10 \times$ larger sample size than McSwain et al. (2009). Our sample includes both cluster and field stars, which differs from earlier studies that focused solely on cluster populations (e.g., McSwain et al. 2009). We found $22 \% \pm 2 \%$ (100/452 cluster and field $\mathrm{Be}$ stars; Table 5) of Be stars experienced a disk-loss or diskrenewal episode within $1 \mathrm{yr}$, which is lower than the composite mean of $51 \% \pm 7 \%(23 / 45$ Be stars) reported by the smaller McSwain et al. (2009) study, although these transient rates do technically agree within $3 \sigma$ of the quoted errors. The rate of diskloss episodes that we observed across our entire sample ( $13 \%$; Table 5) is similar to the fraction of dissipating or small disks inferred from the IR photometric study of Granada et al. (2018). We do caution that Be stars have been found to vary over periods of many years, and our one-year survey may not have captured the full transition period for many of these stars. Indeed, Figure 10 illustrates that many of our nontransient sources exhibit variability, some of which could be revealed to be the onset of transient events given higher photometric accuracy and/or additional epochs of observations.

We note that the compilation of transient Be fractions from McSwain et al. $(2008,2009)$ was computed from four years (NGC 3766) and two years (Collinder 272, Jogg 16, IC 2581, 

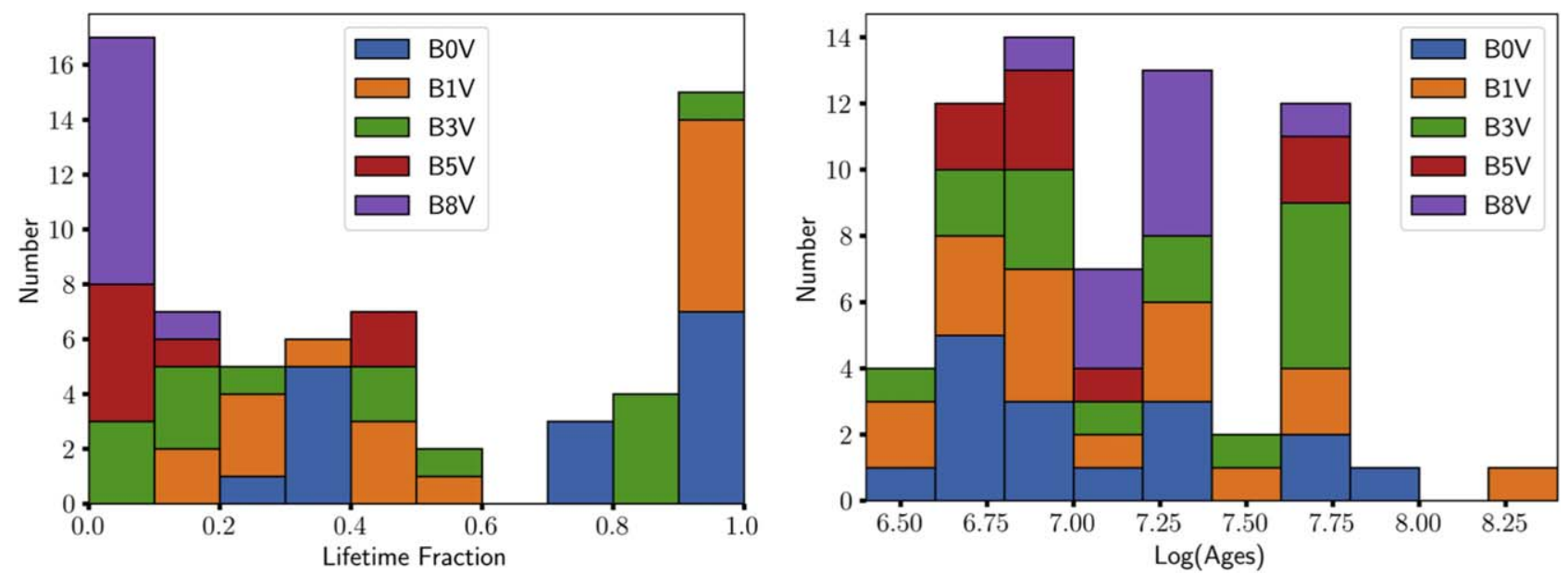

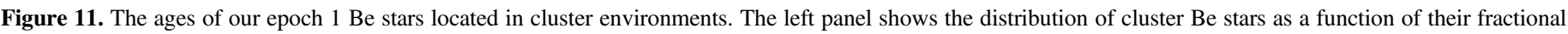

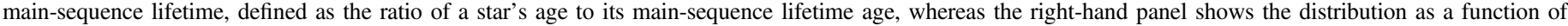
cluster age. Two cluster stars were omitted from this figure as they lacked robust pre-existing age estimates.

NGC 3293, NGC 4755, NGC 6231, and NGC 6664) of observations, so it is not clear how much the different durations of these surveys affect these statistical comparisons. If we make the simple assumption that, to first order, the Be transient fraction over several years scales linearly by a rate set by the duration of observations, the resultant yearly transient $\mathrm{Be}$ fractions are remarkably consistent. Namely, the $22 \% \pm$ $2 \% \mathrm{yr}^{-1}$ we determined from our present study is consistent with that derived from NGC $3766\left(17.3 \% \pm 3 \% \mathrm{yr}^{-1}\right.$; McSwain et al. 2008) and the remaining six clusters in McSwain et al. (2009); $(20.5 \% \pm 4.5 \%)$. Since the rate of diskoutburst events has been noted to be more prevalent in earlytype stars compared to midtype Be stars (Labadie-Bartz et al. 2018), it could be interesting to further build up statistics on the rate of the transient $\mathrm{Be}$ fraction as a function of spectral type, to see if a similar trend emerges.

Finally, we remark that our detection of modestly more diskloss events (57; Table 5) compared to disk-renewal events (43; Table 5) is unexpected given the duration of our sample (1 yr) and previous reports that the disk dissipation phase can last for $\sim 2 \times$ the typical timescale for disk build-up phases (Vieira et al. 2017; Labadie-Bartz et al. 2018). If disk dissipation is a slow process, we would expect to preferentially detect the more rapid disk renewal events, which is the opposite of what we observe. We caution that our adoption of conservative 2-CD criteria for the presence of a disk, requiring a $3 \sigma$ elevation above $\mathrm{H} \alpha$ photospheric levels, could be introducing an observational bias in our data that misclassifies disks decreasing in mass as disk-loss events, and conversely underestimates small-scale disk-renewal events.

\subsection{Age Dependence of the Be Phenomenon}

We explored how Be stars identified in cluster environments depended on cluster age and fractional main-sequence lifetimes in Section 3.5. Interpreting trends in these data first requires a discussion of the known limitations of our data set. Fundamentally, our analysis is enabled by characterization of the fundamental stellar parameters of our data set by use of the BEAST Bayesian toolset. However, as noted in Section 3.2, we do expect our spectral subtyping to have uncertainties of 1-2 subtypes. We see likely byproducts of these uncertainties in
Figure 11. For example, the presence of early subtype Be stars in clusters >30 Myr old could imply these clusters have experienced multiple episodes of star formation and/or be a reflection that the spectral subtypes assigned to this population are incorrect by 1-2 subtypes. Similarly, we noted in Section 3.5 that we reassigned 12 cluster stars to have a fractional mainsequence lifetime of 1 (from $>1$ ), which was a likely byproduct of small mismatches in our spectral subtyping.

With this caveat in mind, we do see several interesting trends appear in Figure 11. First, we observe a clear population of Be stars at early fractional main-sequence lifetimes. This supports the idea that a subset of classical Be stars must emerge on the ZAMS as rapid rotators, as suggested by Wisniewski et al. (2007). Although the enhancement in the number of early-type Be stars observed at late fractional main-sequence lifetimes could support the idea that the Be phenomenon is enhanced with evolutionary age (Fabregat \& Torrejón 2000; Martayan et al. 2010), we caution that we do not have sufficient statistics with our existing M31 data set to uniformly sample how the frequency of early-type Be stars changes as a function of fractional main-sequence lifetime.

\subsection{Be Fraction and Metallicity}

Many studies of Galactic, LMC, and SMC Be stars have found that the fractional Be content of an environment is related inversely to its metallicity (Maeder et al. 1999; Martayan et al. 2006, 2010; Wisniewski \& Bjorkman 2006; Iqbal \& Keller 2013). The reported amplitude of this trend varies substantially in the literature, likely due to differences in completeness, filter systems, disparate stellar ages, and nonuniform ranges of spectral types among the data sets. This trend has been suggested to arise from differences in the average rotation rates of stars in these environments, with low metallicity environments having a larger population of rapid rotators (e.g., Maeder \& Meynet 2000, 2001; Martayan et al. $2007 b)$. Since the present day metallicity of M31 is approximately $1.5 \times$ solar (Clayton et al. 2015), our data set allows us to explore this trend on an extended metallicity range.

The spectral type dependence of the fractional Be content has been extensively studied in the Galaxy, LMC, and SMC (see, e.g., Zorec \& Briot 1997; McSwain \& Gies 2005b; Wisniewski \& Bjorkman 2006; Mathew et al. 2008; Martayan et al. 2010; 


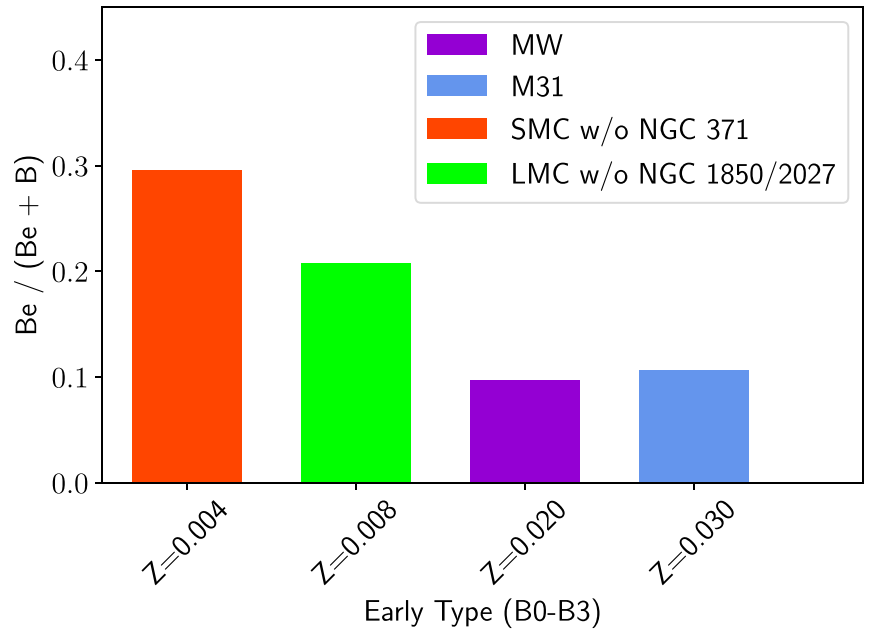

Figure 12. The fractional Be content of early-type (B0-B3) stars is shown as a function of metallicity. We adopted metallicities $(Z)$ of $0.030(1.5 \times$ solar $)$ for M31, $Z=0.020$ for the Milky Way, $Z=0.008$ for the LMC, and $Z=0.004$ for the SMC. The LMC and SMC data are taken from Table 6 of Wisniewski \& Bjorkman (2006), which includes data from Maeder et al. (1999) and Keller et al. (1999); we chose to omit several clusters with abnormally large samples sizes that would otherwise bias our sample averages (e.g., NGC 371 in the SMC; NGC 1850 and NGC 2027 in the LMC). We did attempt to include additional data (e.g., Martayan et al. 2007a), however discrepancies in the available online data prevented uniform inclusion of these data. Our Milky Way data were obtained from McSwain \& Gies (2005b) and Wisniewski \& Bjorkman (2006); we assigned spectral subtypes to these data based on criteria compiled in Martayan et al. (2010).

Rivinius et al. 2013). Although the decrease in percentage of Be stars with spectral type that we observed in M31 (Figure 9) is generally consistent with that reported in the literature in other environments, significant attention must be paid to factors such as sample size, completeness, and the methodology used to quantify Be stars in each study.

The number of single epoch Be stars we identified for statistical analysis in our M31 sample ( 250 Be stars; Table 4$)$, is $1.6 \times$ larger than the full Galactic sample used by McSwain $\&$ Gies (2005b), $2.0 \times$ larger than the full Galactic sample used by Mathew et al. (2008), 1.7× larger than the slitless SMC sample of Martayan et al. (2010), 1.2× larger than the photometric SMC sample of Wisniewski \& Bjorkman (2006), and $1.2 \times$ larger than the photometric LMC sample of Wisniewski \& Bjorkman (2006). However, despite our large sample size, the total number of main-sequence B-type stars (\# normal $\mathrm{B}+\# \mathrm{Be}$ ) that we found as a function of spectral subtype (Table 4) is flat through the mid- to late-B-type stars. This deviation from that expected based on a nominal IMF distribution indicates our M31 sample is incomplete at mid- to late- spectral subtypes, similar to that reported for all SMC and LMC studies (see, e.g., Wisniewski \& Bjorkman 2006; Martayan et al. 2010).

If we limit the detailed comparison of our M31 results with the literature to our earliest spectral subtypes, where completeness is less of an issue, we do find some interesting results. Figure 12 depicts the fractional Be content for early-type (B0B3) stars as a function of metallicity, using Galactic Be star data from McSwain \& Gies (2005b), LMC and SMC Be star data from Wisniewski \& Bjorkman (2006), Maeder et al. (1999), and Keller et al. (1999), and M31 Be star data from the present study. The fractional Be content we observe in M31 is lower than that observed in the SMC or LMC, in agreement with metallicity trends previously noted in the literature

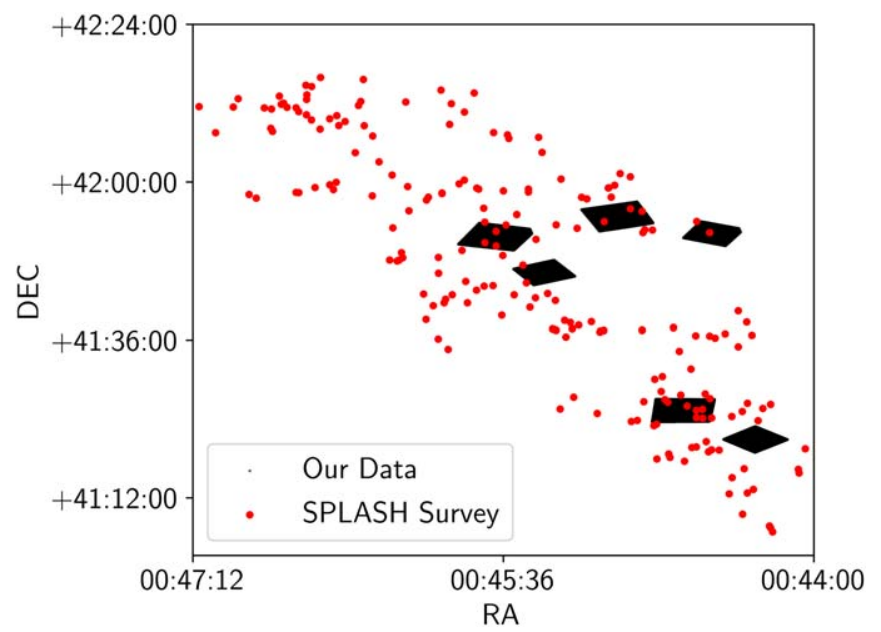

Figure 13. A plot of the R.A. and decl. of stars listed in the SPLASH H $\alpha$ survey as emission stars with stars in our pointings in black.

(Maeder et al. 1999; Martayan et al. 2006, 2010; Wisniewski \& Bjorkman 2006; Iqbal \& Keller 2013). While Be stars are more prevalent in the SMC than in M31 for the earliest subtypes by a factor of $2.8 \times$, this ratio is still lower than the $3-5 \times$ enhancement between the SMC and the Milky Way reported by Martayan et al. (2010). By contrast, our data indicate no clear change in the frequency of early-type Be stars in M31 and the Milky Way, despite their different metallicity. Since the current star formation rate of M31 is lower than that of the Milky Way (Yin et al. 2009; Robitaille \& Whitney 2010; Lewis et al. 2015), it is possible that our results are a reflection that the Be phenomenon is enhanced by evolutionary age as stars progress through their main-sequence evolutionary lifetimes.

\subsection{Comparison with Previous M31 Studies}

The SPLASH survey (Dorman et al. 2012) conducted a Keck II/DEIMOS optical spectroscopic survey of $~ 5000$ isolated stars in M31, including regions observed by the PHAT survey. The SPLASH survey took into account the amount of crowding present during targeting to reduce the role of nearby neighbor contamination, and targeted sources brighter than $\mathrm{F} 814 \mathrm{~W}=22$ or $\mathrm{F} 475 \mathrm{~W}=24$ (Prichard et al. 2017). The survey identified a total of 146 main-sequence Be stars that overlapped with the PHAT footprint and determined a net fraction Be content (\# $\mathrm{Be} /(\#$ normal $\mathrm{B}+\# \mathrm{Be}$ )) of $\sim 12 \%$ (Prichard et al. 2017).

We identified a total of 14 emission line stars in the SPLASH $\mathrm{H} \alpha$ emission survey that overlapped with our HST footprint. These include 10 stars classified as Be stars, 2 classified as RHeB, 1 classified as AGB, and 1 classified as T-MS (Figure 13). SPLASH utilized $0 . " 8$ wide slitlets on Keck/DEIMOS. To investigate the potential role of source confusion and contamination in these spectra, we computed the number of sources in our HST footprint that fell within the Keck/DEIMOS aperture size for each of the 10 Be stars identified by Prichard et al. (2017) that fell within our FOV. We found 24 HST-detected sources in total were present within the Keck aperture for these 10 Be stars, and provide a summary of each of the HST-detected sources that fell within these Keck/DEIMOS apertures in Table 7. Most of these HSTdetected sources had BEAST-derived stellar parameters that were inconsistent with them being main-sequence B-type stars: 7 HSTdetected sources had $T_{\text {eff }}$ more consistent with O-type stars and 14 
Table 7

Be Star Match Data

\begin{tabular}{|c|c|c|c|c|c|c|c|c|c|c|}
\hline $\begin{array}{l}\text { SPLASH Sur- } \\
\text { vey Index }\end{array}$ & Pointing & R.A. & Decl. & F475W & $\begin{array}{c}\mathrm{F} 625 \mathrm{~W} \\
-\mathrm{F} 658 \mathrm{~N}\end{array}$ & $\begin{array}{c}\text { Exceeds } 3 \sigma \\
\text { Expected }\end{array}$ & $\begin{array}{c}\mathrm{F} 475 \mathrm{~W} \\
-\mathrm{F} 814 \mathrm{~W}\end{array}$ & Temperature $(\mathrm{K})$ & $\log (g)_{50}$ & $\log (g)_{84}$ \\
\hline 20276 & 6 & $00: 44: 36.4$ & $+41: 25: 19.5$ & 19.37 & 0.22 & Unknown & 0.16 & 37103 & 3.62 & 3.72 \\
\hline 20276 & 6 & $00: 44: 36.4$ & $+41: 25: 19.2$ & 23.80 & 0.32 & True & 2.04 & 23061 & 3.09 & 3.58 \\
\hline 21673 & 6 & $00: 44: 34.4$ & $+41: 24: 06.4$ & 20.62 & 0.15 & Unknown & -0.07 & 42266 & 4.18 & 4.28 \\
\hline 21673 & 6 & $00: 44: 34.4$ & $+41: 24: 06.1$ & 24.82 & -0.06 & False & 0.62 & 9096 & 3.52 & 3.65 \\
\hline 21673 & 6 & $00: 44: 34.4$ & $+41: 24: 06.2$ & 26.11 & -0.14 & False & 1.62 & 13293 & 4.11 & 4.32 \\
\hline 21767 & 6 & $00: 44: 34.4$ & $+41: 25: 28.8$ & 19.75 & 0.86 & Unknown & 0.46 & 49538 & 3.99 & 4.10 \\
\hline 22397 & 6 & $00: 44: 45.2$ & $+41: 26: 33.8$ & 21.31 & 0.28 & True & 0.53 & 29677 & 3.61 & 3.83 \\
\hline 22397 & 6 & $00: 44: 45.1$ & $+41: 26: 34.0$ & 25.48 & 0.11 & False & 1.09 & 5385 & 2.69 & 2.89 \\
\hline 22397 & 6 & $00: 44: 45.2$ & $+41: 26: 33.6$ & 25.05 & -0.43 & False & 0.57 & 10796 & 3.74 & 3.95 \\
\hline 22397 & 6 & $00: 44: 45.2$ & $+41: 26: 33.6$ & 25.72 & 0.45 & False & 1.52 & 4823 & 2.48 & 2.68 \\
\hline 22760 & 2 & $00: 44: 32.2$ & $+41: 52: 17.6$ & 22.09 & 0.19 & False & 0.78 & 23175 & 3.41 & 3.60 \\
\hline 22760 & 2 & $00: 44: 32.2$ & $+41: 52: 17.3$ & 26.29 & $\ldots$ & False & 1.83 & 4913 & 2.55 & 2.77 \\
\hline 22776 & 4 & $00: 45: 38.3$ & $+41: 52: 28.5$ & 21.03 & 0.14 & Unknown & 0.09 & 34252 & 4.00 & 4.12 \\
\hline 22776 & 4 & $00: 45: 38.3$ & $+41: 52: 28.1$ & 25.95 & -1.46 & False & 1.81 & 4946 & 2.50 & 2.67 \\
\hline 22859 & 5 & $00: 45: 04.8$ & $+41: 53: 59.3$ & 21.09 & 0.84 & Unknown & 0.60 & 46804 & 4.08 & 4.25 \\
\hline 22859 & 5 & $00: 45: 04.8$ & $+41: 53: 59.0$ & 26.34 & -0.33 & False & 2.05 & 4717 & 2.42 & 2.60 \\
\hline 23050 & 5 & $00: 44: 53.1$ & $+41: 55: 32.4$ & 20.98 & 0.21 & Unknown & 0.58 & 38616 & 3.88 & 4.08 \\
\hline 23073 & 5 & $00: 44: 56.7$ & $+41: 55: 54.9$ & 22.85 & 0.41 & True & 0.15 & 15174 & 3.59 & 3.70 \\
\hline 23073 & 5 & $00: 44: 56.7$ & $+41: 55: 54.9$ & 26.24 & 0.00 & False & 1.86 & 5938 & 2.72 & 2.97 \\
\hline
\end{tabular}

Note. Shown here are the relevant data for the stars within the aperture radius of stars in the SPLASH survey. $\log (g)_{x}$ is the $x^{\prime}$ th percentile of the $\log (g)$ posterior probability distribution and "Exceeds $3 \sigma$ Expected" denotes whether the star meets the emission line criteria outlined in Section 3. Some stars in our survey were assigned identical R.A./decl. coordinates due source confusion in the PHAT catalog.

$H S T$-detected sources had $\log (g)$ values more consistent with post-main-sequence giants. As seen in Table 7, each SPLASH Be star was matched with one source having a bright $\mathrm{F} 475 \mathrm{~W}$ magnitude, along with several less luminous stars. It is therefore possible that the $\mathrm{H} \alpha$ EWs reported by Prichard et al. (2017) have slightly elevated (or suppressed) signal from these fainter neighbors that could lead to spurious detections of Be stars in the survey. We note that none of the stars that we identified as Be stars in the analysis of our HST data were matched with Be stars identified by the SPLASH survey.

Given the clear dependence of the Be phenomenon as a function of spectral subtype that we see in our M31 sample (Figure 9 and Table 4) and the shallower depth of source brightnesses probed by the SPLASH survey, we caution that the global (spanning all spectral subtypes) M31 Be fraction of $\sim 12 \%$ cited by Prichard et al. (2017) is strongly biased by overweighting contributions from the earliest B subtypes.

In a broader context, we noted in Section 3.5 that our HST survey also likely suffered from source confusion, especially in the densest cluster regions of M31. If the effects of source confusion serve to produce a greater frequency of false-positive detections of classical Be stars, as seems possible from our overlapping $H S T$ coverage of the SPLASH $\mathrm{H} \alpha$ footprint, this would imply that the Be fractions we derived in our study could be similarly affected by false-positive detections, especially in the densest stellar environments.

\section{Conclusions}

We have presented results from a 12 orbit, two epoch $H S T$ $\mathrm{H} \alpha$ emission line survey of the Andromeda galaxy that overlapped with the footprint of the HST/PHAT survey, focusing on the classical Be star content of the galaxy. Roughly 2 million sources were detected in each epoch; after applying criteria to isolate the classical Be star population in this sample, we found:

1. In total, 552 (542) classical Be stars and 8429 (8556) normal B-type stars were identified in epoch \# 1 (epoch \# 2). The overall fractional Be content (\# Be/(\# normal B + $\# \mathrm{Be})$ ) we found was $6.15 \% \pm 0.26 \%(5.96 \% \pm 0.25 \%)$ in epoch \# 1 (epoch \# 2). These overall rates are lower than that reported by the Keck II/DEIMOS-based SPLASH survey ( 12\%, Prichard et al. 2017). We suggest this discrepancy arises from the selection criteria for the SPLASH survey overweighting contributions from the earliest B subtype stars, which are more likely to exhibit the Be phenomenon, as well as potential source confusion in SPLASH.

2. The fractional Be content decreased with spectral subtype from $\sim 23.6 \% \pm 2.0 \% \quad(\sim 23.9 \% \pm 2.0 \%)$ for B0-type stars to $\sim 3.1 \% \pm 0.26 \%(\sim 3.4 \% \pm 0.35 \%)$ for B8-type stars in epoch \# 1 (epoch \# 2). We caution that our latertype Be population likely suffers from incompleteness. 
Table 8

Source Catalog

\begin{tabular}{|c|c|c|c|c|c|c|c|c|c|c|c|c|c|c|c|}
\hline Pointing & Epoch & Index & R.A. & Decl. & $\mathrm{F} 475 \mathrm{~W}$ & $\mathrm{~F} 625 \mathrm{~W}$ & $\mathrm{~F} 658 \mathrm{~N}$ & $\mathrm{~F} 814 \mathrm{~W}$ & $\log (T)_{\text {eff }}$ & $\log (g)_{50}$ & $\log (g)_{84}$ & Cluster & Be Star? & $\begin{array}{l}\text { Spec } \\
\text { Type }\end{array}$ & Var? \\
\hline 1 & 1 & 1907281 & 11.369 & 41.785 & $21.309 \pm 0.003$ & $21.242 \pm 0.008$ & $21.284 \pm 0.042$ & $21.175 \pm 0.004$ & 4.505 & 3.949 & 4.104 & 0 & False & B0V & $\overline{\text { BSTAR }}$ \\
\hline 1 & 1 & 1022497 & 11.371 & 41.76 & $21.733 \pm 0.004$ & $21.361 \pm 0.008$ & $21.567 \pm 0.043$ & $21.248 \pm 0.005$ & 4.496 & 3.827 & 3.983 & 0 & False & $\mathrm{B} 0 \mathrm{~V}$ & BSTAR \\
\hline 1 & 1 & 1907276 & 11.383 & 41.774 & $21.244 \pm 0.003$ & $21.170 \pm 0.008$ & $21.135 \pm 0.032$ & $21.078 \pm 0.004$ & 4.396 & 3.562 & 3.781 & 0 & False & B1V & BSTAR \\
\hline$\cdots$ & $\ldots$ & $\ldots$ & $\ldots$ & $\ldots$ & $\ldots$ & $\ldots$ & $\ldots$ & $\ldots$ & $\ldots$ & $\ldots$ & $\ldots$ & $\cdots$ & $\ldots$ & $\ldots$ & $\ldots$ \\
\hline
\end{tabular}

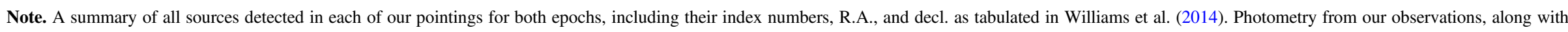

A the PHAT survey as compiled in Williams et al. (2014) are provided for each source. All photometry errors are cited at the $1 \sigma$ level. The quoted $T_{\text {eff }}$ and Log $(g)$ values were computed via the

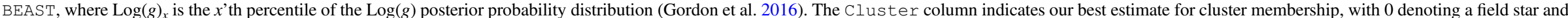

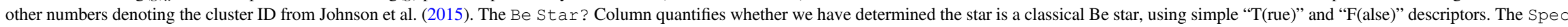

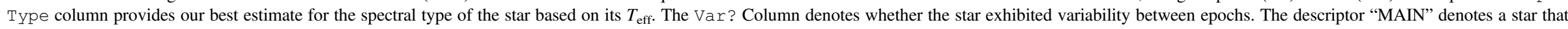

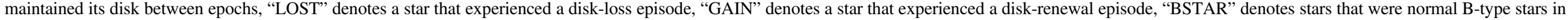
both epochs, and "UNKWN" is a star that was detected in only one of the epochs.

(This table is available in its entirety in machine-readable form.) 
3. The fractional Be content we determined for B0-B3-type stars in M31 is lower than that observed for the same subset of spectral type objects in the SMC and LMC. This provides confirmation that the fractional Be content in more metal-rich environments (the Milky Way and $\sim 1.5 \times$ solar M31) is lower than that observed in metalpoor environments (LMC and SMC). We found that Be stars are more prevalent in the SMC than in M31 for the earliest subtypes by a factor of $2.8 \times$; this ratio is lower than the 3-5× enhancement between the SMC and Milky Way reported by Martayan et al. (2010).

4. We observed a clear population of Be stars in cluster environments at early fractional main-sequence lifetimes. This supports the idea that a subset of classical Be stars emerge onto the ZAMS as rapid rotators. Since M31 has a lower star formation rate compared to the Milky Way, the lack of an observed change in the frequency of earlytype Be stars between these two galaxies, despite their $1.5 \times$ metallicity difference, may reflect that the $\mathrm{Be}$ phenomenon is also enhanced by evolutionary age.

5. In our sample, $22 \% \pm 2 \%$ of stars exhibited evidence of either a disk-loss or disk-regeneration episode in the $\sim 1$ year interval between our two epochs of observations. If we assume that the Be transient fraction over timescales of several years scales linearly by a rate set by the duration of observations, the $22 \% \pm 2 \% \mathrm{yr}^{-1}$ transient rate we observed in M31 is consistent with that derived from NGC $3766\left(17.3 \% \pm 3 \% \mathrm{yr}^{-1}\right.$; McSwain et al. 2008) and the remaining six clusters in McSwain et al. (2009); $(20.5 \% \pm 4.5 \%)$. We observed a similar number of disk-loss events (57) as disk-renewal events (43), which was unexpected as disk dissipation timescales can be $\sim 2 \times$ the typical timescales for disk build-up phases (Vieira et al. 2017; Labadie-Bartz et al. 2018).

J.W. thanks Karen Bjorkman for stimulating discussions of the classical Be phenomenon and this paper. This work was supported by STScI grants for GO-13857, and by NSF-AST 1412110. This work has made use of the BeSS database, operated at LESIA, Observatoire de Meudon, France: http:// basebe.obspm.fr.

Software: DOLPHOT (Dolphin 2000), pysynphot (STScI Development Team 2013), BEAST (Gordon et al. 2016), matplotlib (ud-Doula et al. 2018), scipy (Virtanen et al. 2019).

\section{Appendix \\ Focus-induced Systematics}

During our basic assessment of the quality of our pipeline photometry products, we noted a -0.1 mag offset between epochs in our $\mathrm{F} 625 \mathrm{~W}$ photometry of pointings 2, 3, 5, and 6 . No such offset were seen in our $\mathrm{F} 658 \mathrm{~N}$ data, or between epochs for pointings 1 and 4. Moreover, we observed clear variations in the shape of the PSF from epoch-to-epoch difference images. This suggests that the photometric offsets we observe are due to $H S T$ focus variations that are not otherwise accounted for in our photometry. It is well known that HST experiences "breathing" over the course of an orbit, causing the distance between the primary and secondary mirror (the "despace") to vary on the order of $\pm 5 \mu \mathrm{m}$ from nominal. This causes corresponding variations in the PSF; when the secondary mirror position is off-nominal (i.e., has a nonzero despace value), light is proportionally less concentrated in the core of the PSF and more concentrated in the first and second Airy rings. Dressel (2012) demonstrated that for WFC3/UVIS F420M the fraction of encircled energy within a 0 ". 15 aperture decreases by over $20 \%$ going from nominal to +5 micron despace.

In most scenarios, any PSF variation induced by focus is not the dominant systematic in HST photometry; most long exposures ( $>\sim 2000 \mathrm{~s}$ ) and co-adds of multiple short exposures are likely to sample the short-term focus variation adequately, and if one uses empirically determined PSFs for photometry or TinyTim (Krist et al. 2011) PSFs at correct focus values, then the effects of focus will most likely be accounted for to within photometric error. As our photometry uses a single model PSF at nominal focus (see e.g., Williams et al. 2014), we are sensitive to focus-induced systematics. It is also worth noting that the focus model shows moderate deviation from measured focus values, and that there are other factors affecting focus that are not accounted for by the model (J. H. van Gorkom 2016, private communication); however, for our purposes we are satisfied that the focus deviations predicted by the model account for the photometric offsets we observe.

We measured the focus for each individual exposure using the HST focus tool, ${ }^{9}$ which implements the focus model described in Cox \& Lallo (2012). For our epoch 1 data, we found a despace value close to nominal for pointings $2,3,5$, and 6 , and between 3 and 4 microns for pointings 1 and 4 . For our epoch 2 data, all pointings had despace values between 3 and 4 microns. To correct our data for this constant offset and ensure the same relative flux calibration is present across our entire data set, we therefore subtracted $0.1 \mathrm{mag}$ from pointings 1 and 4 in epoch 1 and pointings $1-6$ in epoch 2 .

\section{ORCID iDs}

M. Peters (1) https://orcid.org/0000-0002-5897-7207

J. P. Wisniewski (i) https://orcid.org/0000-0001-9209-1808

B. F. Williams (iD https://orcid.org/0000-0002-7502-0597

J. R. Lomax (iD https://orcid.org/0000-0001-8470-0853

L. C. Johnson (iD https://orcid.org/0000-0001-6421-0953

T. A. A. Sigut (1D https://orcid.org/0000-0002-0803-8615

J. J. Dalcanton (iD https://orcid.org/0000-0002-1264-2006

\section{References}

Baade, D., Pigulski, A., Rivinius, T., et al. 2018, A\&A, 610, A70 Baade, D., Rivinius, T., Pigulski, A., et al. 2016, A\&A, 588, A56 Carciofi, A. C., Bjorkman, J. E., Otero, S. A., et al. 2012, ApJL, 744, L15 Cassinelli, J. P., Brown, J. C., Maheswaran, M., Miller, N. A., \& Telfer, D. C. 2002, ApJ, 578, 951

Castelli, F., \& Kurucz, R. L. 2004, in Proc. IAU Symp. 210, Modelling of Stellar Atmospheres, ed. N. Piskunov, W. W. Weiss, \& D. F. Gray (Cambridge: Cambridge Univ. Press), A20

Clayton, G. C., Gordon, K. D., Bianchi, L. C., et al. 2015, ApJ, 815, 14

Cox, C., \& Lallo, M. 2012, Proc. SPIE, 8442, 844237

Dalcanton, J. J., Williams, B. F., Lang, D., et al. 2012, ApJS, 200, 18

Dolphin, A. E. 2000, PASP, 112, 1383

Dorman, C. E., Guhathakurta, P., Fardal, M. A., et al. 2012, ApJ, 752, 147

Draper, Z. H., Wisniewski, J. P., Bjorkman, K. S., et al. 2014, ApJ, 786, 120

Dressel, L. 2012, Breathing, Position Drift, and PSF Variations on the UVIS

Detector, Instrument Science Report, WFC3 2012-14

Fabregat, J., \& Torrejón, J. M. 2000, A\&A, 357, 451

Georgy, C., Ekström, S., Granada, A., et al. 2013, A\&A, 553, A24

Gordon, K. D., Fouesneau, M., Arab, H., et al. 2016, ApJ, 826, 104

Granada, A., Jones, C. E., Sigut, T. A. A., et al. 2018, AJ, 155, 50

\footnotetext{
http://focustool.stsci.edu/cgi-bin/control.py
} 
Grebel, E. K., Richtler, T., \& de Boer, K. S. 1992, A\&A, 254, L5

Grundstrom, E. D., McSwain, M. V., Aragona, C., et al. 2011, BSRSL, 80, 371

Haubois, X., Carciofi, A. C., Rivinius, T., Okazaki, A. T., \& Bjorkman, J. E. 2012, ApJ, 756, 156

Hummel, W., \& Vrancken, M. 2000, A\&A, 359, 1075

Iqbal, S., \& Keller, S. C. 2013, MNRAS, 435, 3103

Jaschek, M., Slettebak, A., \& Jaschek, C. 1981, BeSN, 4, 9

Johnson, L. C., Seth, A. C., Dalcanton, J. J., et al. 2015, ApJ, 802, 127

Johnson, L. C., Seth, A. C., Dalcanton, J. J., et al. 2016, ApJ, 827, 33

Keller, S. C., Wood, P. R., \& Bessell, M. S. 1999, A\&AS, 134, 489

Krist, J. E., Hook, R. N., \& Stoehr, F. 2011, Proc. SPIE, 8127, 81270J

Kroupa, P. 2001, MNRAS, 322, 231

Labadie-Bartz, J., Chojnowski, S. D., Whelan, D. G., et al. 2018, AJ, 155, 53

Labadie-Bartz, J., Pepper, J., McSwain, M. V., et al. 2017, AJ, 153, 252

Lee, U., Osaki, Y., \& Saio, H. 1991, MNRAS, 250, 432

Lewis, A. R., Dolphin, A. E., Dalcanton, J. J., et al. 2015, ApJ, 805, 183

Maeder, A., Grebel, E. K., \& Mermilliod, J.-C. 1999, A\&A, 346, 459

Maeder, A., \& Meynet, G. 2000, ARA\&A, 38, 143

Maeder, A., \& Meynet, G. 2001, A\&A, 373, 555

Martayan, C., Baade, D., \& Fabregat, J. 2010, A\&A, 509, A11

Martayan, C., Floquet, M., Hubert, A. M., et al. 2007a, A\&A, 472, 577

Martayan, C., Frémat, Y., Hubert, A.-M., et al. 2006, A\&A, 452, 273

Martayan, C., Frémat, Y., Hubert, A.-M., et al. 2007b, A\&A, 462, 683

Mathew, B., Subramaniam, A., \& Bhatt, B. C. 2008, MNRAS, 388, 1879

McSwain, M. V., \& Gies, D. R. 2005a, ApJ, 622, 1052

McSwain, M. V., \& Gies, D. R. 2005b, ApJS, 161, 118

McSwain, M. V., Huang, W., \& Gies, D. R. 2009, ApJ, 700, 1216

McSwain, M. V., Huang, W., Gies, D. R., Grundstrom, E. D., \& Townsend, R. H. D. 2008, ApJ, 672, 590

Meilland, A., Stee, P., Vannier, M., et al. 2007, A\&A, 464, 59

Mennickent, R. E., Vogt, N., Barrera, L. H., Covarrubias, R., \& Ramirez, A. 1994, A\&AS, 106, 427

Meynet, G., \& Maeder, A. 2000, A\&A, 361, 101

Neiner, C., de Batz, B., Cochard, F., et al. 2011, AJ, 142, 149

Neiner, C., Floquet, M., Samadi, R., et al. 2012, A\&A, 546, A47

Neiner, C., Hubert, A.-M., Floquet, M., et al. 2002, A\&A, 388, 899

Porter, J. M., \& Rivinius, T. 2003, PASP, 115, 1153
Prichard, L. J., Guhathakurta, P., Hamren, K. M., et al. 2017, MNRAS, 465, 4180

Rivinius, T., Baade, D., Stefl, S., et al. 1998, A\&A, 333, 125

Rivinius, T., Baade, D., \& Štefl, S. 2003, A\&A, 411, 229

Rivinius, T., Carciofi, A. C., \& Martayan, C. 2013, A\&ARv, 21, 69

Rivinius, T., Štefl, S., \& Baade, D. 2006, A\&A, 459, 137

Robitaille, T. P., \& Whitney, B. A. 2010, ApJL, 710, L11

Rogers, T. M., Lin, D. N. C., McElwaine, J. N., \& Lau, H. H. B. 2013, ApJ, 772,21

Silaj, J., Jones, C. E., Sigut, T. A. A., \& Tycner, C. 2014, ApJ, 795, 82

Silaj, J. M. 2014, PhD thesis, Univ. Western Ontario

Slettebak, A., Dollins, G. W. I., \& Truax, R. 1992, ApJS, 81, 335

Struve, O. 1931, ApJ, 73, 94

STScI Development Team 2013, Pysynphot: Synthetic Photometry Software Package, Astrophysics Source Code Library, ascl:1303.023

ud-Doula, A., Owocki, S. P., \& Kee, N. D. 2018, MNRAS, 478, 3049

Ud-Doula, A., Owocki, S. P., \& Townsend, R. H. D. 2008, MNRAS, 385, 97

Underhill, A. B., Divan, L., Prevot-Burnichon, M.-L., \& Doazan, V. 1979, MNRAS, 189,601

Veyette, M. J., Williams, B. F., Dalcanton, J. J., et al. 2014, ApJ, 792, 121

Vieira, R. G., Carciofi, A. C., Bjorkman, J. E., et al. 2017, MNRAS, 464, 3071

Virtanen, P., Gommers, R., Oliphant, T. E., et al. 2019, arXiv:1907.10121

Wade, G. A., Petit, V., Grunhut, J., \& Neiner, C. 2014, in Bright Emissaries: Be Stars as Messengers of Star-Disk Physics, ed. T. A. A. Sigut \& C. E. Jones (San Fransisco, CA: ASP), 207

Wheelwright, H. E., Bjorkman, J. E., Oudmaijer, R. D., et al. 2012, MNRAS, 423, L11

Williams, B. F., Lang, D., Dalcanton, J. J., et al. 2014, ApJS, 215, 9

Williams, B. F., Olsen, K., Khan, R., Pirone, D., \& Rosema, K. 2018, ApJS, 236,4

Wisniewski, J. P., \& Bjorkman, K. S. 2006, ApJ, 652, 458

Wisniewski, J. P., Bjorkman, K. S., Magalhães, A. M., et al. 2007, ApJ, 671,2040

Wisniewski, J. P., Draper, Z. H., Bjorkman, K. S., et al. 2010, ApJ, 709, 1306

Yin, J., Hou, J. L., Prantzos, N., et al. 2009, A\&A, 505, 497

Zorec, J., \& Briot, D. 1997, A\&A, 318, 443 